\title{
NEW HYBRIDIZED MIXED METHODS FOR LINEAR ELASTICITY AND OPTIMAL MULTILEVEL SOLVERS
}

\author{
SHIHUA GONG, SHUONAN WU, AND JINCHAO XU
}

\begin{abstract}
In this paper, we present a family of new mixed finite element methods for linear elasticity for both spatial dimensions $n=2,3$, which yields a conforming and strongly symmetric approximation for stress. Applying $\mathcal{P}_{k+1}-\mathcal{P}_{k}$ as the local approximation for the stress and displacement, the mixed methods achieve the optimal order of convergence for both the stress and displacement when $k \geq n$. For the lower order case $(n-2 \leq k<n)$, the stability and convergence still hold on some special grids. The proposed mixed methods are efficiently implemented by hybridization, which imposes the inter-element normal continuity of the stress by a Lagrange multiplier. Then, we develop and analyze multilevel solvers for the Schur complement of the hybridized system in the two dimensional case. Provided that no nearly singular vertex on the grids, the proposed solvers are proved to be uniformly convergent with respect to both the grid size and Poisson's ratio. Numerical experiments are provided to validate our theoretical results.
\end{abstract}

\section{INTRODUCTION}

The mixed finite element methods are popular in solid mechanics since they avoid locking and provide a straightforward approximation for stress. The conforming mixed methods based on the classical Hellinger-Reissner variational formulation requires finite element space for the stress in $H(\operatorname{div} ; \mathbb{S})$, the space of symmetric matrix-valued fields, which are square integrable with square integrable divergence. In the meantime, the discrete space for the stress must be compatible with that for the displacement, which is a subspace of the vector-valued $L^{2}$ space. However, the construction of such stable pairs using polynomial shape functions is very challenging.

To overcome this difficulty, the earliest works adopted composite element techniques (cf. [39, 7]). The composite element methods approximate the displacement in one grid while approximating the stress in the refined grid. Due to the difficulties in keeping the symmetry and conformity at the same time, some compromised methods that relax one of the two requirements have been developed. The first category of such methods (cf. [2, 45, 46, 8, 14, 23, 30]) weakly imposes stress symmetry, while maintaining exact $H$ (div) conformity. These methods introduce the Lagrange multiplier, approximating the non-symmetric part of the displacement

2010 Mathematics Subject Classification. 65N30, 65N55.

The work of the first and third authors was supported in part by National Natural Science Foundation of China (NSFC) (Grant No. 91430215, 41390452) and by Beijing International Center for Mathematical Research of Peking University, China.

The work of the second and third authors was supported in part by the DOE Grant desc0009249 as part of the Collaboratory on Mathematics for Mesoscopic Modeling of Materials and by DOE Grant de-sc0014400 and NSF Grant DMS-1522615. 
gradient while enforcing stress symmetry weakly. The second category of such methods (cf. [12, 13, 34, 43, 55, 56, 26]) relaxes the conformity constraints while keeping the symmetry strongly.

In [11, Arnold and Winther proposed the first family of mixed finite element methods in two dimension (2D), which yields the symmetric and conforming approximation for the stress. Since then, many stable mixed elements have been constructed, see [4, 5, 1. However, the shape function spaces of these elements, using incomplete polynomials, are quite complicated. In [35, 36], $\mathrm{Hu}$ and Zhang constructed a family of mixed finite elements with conforming and symmetric stress approximation in a unified fashion on simplex grids for spatial dimension $n=2,3$. The degrees of the polynomials to approximate the stress and displacement match reasonably and naturally, by which these elements also achieve the optimal order of convergence. The generalizations or variants of Hu-Zhang's finite elements can be found in [32, 33, 37.

Both families of the conforming elements above are subject to continuity constraints at the element vertices, which is not natural for $H$ (div) conformity and prohibits techniques like hybridization that are usually available for the mixed method. One feature of our methods is to relax the continuity at the element vertices using the full $C^{\text {div }}-\mathcal{P}_{k+1}$ space for the stress

$$
\Sigma_{h, k+1}=\left\{\boldsymbol{\tau} \in H(\operatorname{div}, \Omega ; \mathbb{S})|\boldsymbol{\tau}|_{K} \in \mathcal{P}_{k+1}(K ; \mathbb{S}) \quad \forall K \in \mathcal{T}_{h}\right\} .
$$

Taking the full $C^{-1}-\mathcal{P}_{k}$ vector-valued space $V_{h, k}$ for the displacement, the stability of $\Sigma_{h, k+1}-V_{h, k}$ follows directly from the results of [35, 36, 32] when $k \geq n$. On some special grids, we can still prove the stability for the lower order pairs when $n-2 \leq k<n$. In the 2D case, it is feasible to construct nodal basis functions for $\Sigma_{h, k+1}$ by geometric analysis at the vertices (cf. [44]). In the 3D case, however, it is complicated to deal with nodal basis functions associated with the vertices or edges. In any case, the dimension of $\Sigma_{h, k+1}$ therefore depends on the singular vertices (cf. [44]) or singular edges of the grids.

Instead of constructing basis functions for $\Sigma_{h, k+1}$, we implement it by hybridization (cf. 6, 24]). In other words, we remove the inter-element continuity of stress and enforce it by the Lagrange multiplier - the piecewise discontinuous polynomial space of degree $k+1$ defined on the edges or faces. The stress and displacement can be eliminated locally in the hybridized mixed system, which results in a linear system solely for the Lagrange multiplier. The resulting multiplier system may have a nontrivial kernel due to the singular vertices or singular edges on the grids but leads to a unique solution of the stress and displacement. Related works on hybridizable methods for elasticity can be found in [52, 28, 47. In 28], a family of nonconforming and hybridizable elements on simplicial grids was developed in both 2D and 3D cases. The hybridizable discontinuous Galerkin (HDG) methods for the linear elasticity were studied in [52, 47].

Another feature of our methods is to develop robust iterative solvers for the Schur complement of the hybridized mixed system in the $2 \mathrm{D}$ case, provided that there is no nearly singular vertex on the grids. The iterative solvers for the hybridized mixed method for the diffusion problem were studied in [27, 29, 22, 42, 41]. Although the methodologies in dealing with the non-nested multilevel finite element spaces and the non-inherited bilinear forms were discussed in these papers for the diffusion problem, two essential distinctions exist for the linear elasticity: (i) some local estimates do not hold on each element, but on the element patch, and (ii) the 
condition number of the multiplier system depends not only on the grid size but also on Poisson's ratio.

To overcome these difficulties, we first establish some local estimates on the element patches by characterizing the inter-element jump of piecewise discontinuous symmetric-matrix-valued polynomials (see Lemma 3.9 and 3.10). We then propose an equivalent norm to the energy norm associated with the multiplier system, which indicates that the multiplier system holds a similar structure with that of the stable discretization $\left(P_{2}-P_{0}\right)$ for the elastic primal formulation (cf. [48]). Thus, capturing the rigid-body motion mode and the weak divergence-free mode simultaneously is the key to developing robust iterative solvers with respect to both the grid size and Poisson's ratio.

The rest of the paper is organized as follows. In the next section, we introduce our mixed finite element methods and prove their stability and convergence. In Section 3, we present the hybridization of the mixed finite element method. We also characterize the kernel of the hybridized mixed system and develop some tools to estimate the norms. In Section 4, we focus on the iterative solvers for the multiplier system. We provide some numerical results in Section 5 and give some concluding remarks in Section 6. Finally, some technical results can be found in the appendix.

\section{Mixed Methods}

In this paper, we consider the following linear elasticity problem with Dirichlet boundary condition

$$
\left\{\begin{aligned}
\mathcal{A} \boldsymbol{\sigma}-\boldsymbol{\epsilon}(u)=0 & \text { in } \Omega, \\
\operatorname{div} \boldsymbol{\sigma}=f & \text { in } \Omega, \\
u=0 & \text { on } \partial \Omega,
\end{aligned}\right.
$$

where $\Omega$ is a polygonal domain in $\mathbb{R}^{n}(n=2,3)$. The displacement and stress are denoted by $u: \Omega \mapsto \mathbb{R}^{n}$ and $\sigma: \Omega \mapsto \mathbb{S}$, respectively. Here, $\mathbb{S}$ represents the space of real symmetric matrices of order $n \times n$. The compliance tensor $\mathcal{A}: \mathbb{S} \mapsto \mathbb{S}$ is defined as

$$
\mathcal{A} \boldsymbol{\sigma}:=\frac{1}{2 \tilde{\mu}}\left(\boldsymbol{\sigma}-\frac{\tilde{\lambda}}{2 \tilde{\mu}+n \tilde{\lambda}} \operatorname{tr}(\boldsymbol{\sigma}) \boldsymbol{I}\right),
$$

where $\tilde{\mu}, \tilde{\lambda}$ are the Lamé constants. Clearly, $\mathcal{A}$ is bounded and symmetric positive definite. The linearized strain tensor is denoted by $\epsilon(u)=\left(\nabla u+(\nabla u)^{T}\right) / 2$.

2.1. Preliminaries. Let $\mathcal{T}_{h}$ be a family of quasi-uniform triangulations (cf. [15]) of $\Omega$. Let $h_{K}$ be the diameter of element $K \in \mathcal{T}_{h}$, and $h=\max _{K} h_{K}$ be the grid diameter of $\mathcal{T}_{h}$. For any $K \in \mathcal{T}_{h}$, the set of all elements that share vertex with $K$ is denoted by $\omega_{K}$. The sets of all faces and nodes of $\mathcal{T}_{h}$ are denoted by $\mathcal{F}_{h}$ and $\mathcal{N}_{h}$, respectively. Moreover, $\mathcal{F}_{h}$ can be divided into two subsets: the boundary faces set $\mathcal{F}_{h}^{\partial}=\mathcal{F}_{h} \cap \partial \Omega$ and the interior faces set $\mathcal{F}_{h}^{i}=\mathcal{F}_{h} \backslash \mathcal{F}_{h}^{\partial}$. The unit normal vector with respect to the face $F$ is represented by $\nu_{F}$.

Let $F \in \mathcal{F}_{h}^{i}$ be the common face of two elements $K^{+}$and $K^{-}$, and $\nu_{F}^{+}$and $\nu_{F}^{-}$ be the unit outward normal vectors on $F$ with respect to $K^{+}$and $K^{-}$, respectively. Then, we define the jump [.] on $F \in \mathcal{F}_{h}^{i}$ for $\boldsymbol{\tau}$ by:

$$
[\boldsymbol{\tau}]_{F}:=\boldsymbol{\tau}_{K^{+}} \nu_{F}^{+}+\boldsymbol{\tau}_{K^{-}} \nu_{F}^{-} .
$$


For $F \in \mathcal{F}_{h}^{\partial}$, we define $[\boldsymbol{\tau}]_{F}:=\boldsymbol{\tau} \nu$, where $\nu$ is the unit outer normal along $\partial \Omega$.

Our notation for the inner products is standard (cf. [15]): For $u, v \in L^{2}(D)$, we write $(u, v)_{D}=\int_{D} u v \mathrm{~d} x$ if $D$ is a subdomain of $\mathbb{R}^{n}$, and $\langle u, v\rangle_{D}=\int_{D} u v \mathrm{~d} s$ if $D$ is a subdomain of $\mathbb{R}^{n-1}$. We neglect the subscript $D$ if $D=\Omega$. To emphasize the mesh-dependent nature of certain integrals, for $\widetilde{\mathcal{T}}_{h} \subset \mathcal{T}_{h}$ and $\widetilde{\mathcal{F}}_{h} \subset \mathcal{F}_{h}$, we define

$$
\left(u_{h}, v_{h}\right)_{\widetilde{\mathcal{T}}_{h}}:=\sum_{K \in \widetilde{\mathcal{T}}_{h}}\left(u_{h}, v_{h}\right)_{K} \quad \text { and } \quad\left\langle\lambda_{h}, \mu_{h}\right\rangle_{\widetilde{\mathcal{F}}_{h}}:=\sum_{F \in \widetilde{\mathcal{F}}_{h}}\left\langle\lambda_{h}, \mu_{h}\right\rangle_{F},
$$

where $u_{h}, v_{h}$ and $\mu_{h}, \lambda_{h}$ are defined on $\widetilde{\mathcal{T}}_{h}$ and $\widetilde{\mathcal{F}}_{h}$, respectively.

Throughout this paper, we shall use letter $C$ to denote a generic positive constant independent of $h$ and the material parameters. Note that $C$ may stand for different values at its various occurrences. The notation $x \lesssim y$ means $x \leq C y$ and $x \simeq y$ means $x \lesssim y \lesssim x$.

The mixed formulation of 2.1$)$ is to find $(\boldsymbol{\sigma}, u) \in \Sigma \times V:=H(\operatorname{div}, \Omega ; \mathbb{S}) \times$ $L^{2}\left(\Omega ; \mathbb{R}^{n}\right)$ such that

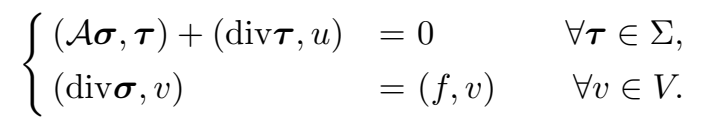

Here, $H(\operatorname{div}, \Omega ; \mathbb{S})$ consists of square-integrable symmetric matrix fields with squareintegrable divergence, and $L^{2}\left(\Omega ; \mathbb{R}^{n}\right)$ is the space of vector-valued functions that are square integrable with the standard $L^{2}$ norm. The corresponding $H$ (div) norm is defined by

$$
\|\boldsymbol{\tau}\|_{H(\operatorname{div})}^{2}:=\|\boldsymbol{\tau}\|_{0}^{2}+\|\operatorname{div} \boldsymbol{\tau}\|_{0}^{2} \quad \forall \boldsymbol{\tau} \in H(\operatorname{div}, \Omega ; \mathbb{S}) .
$$

We take the discrete stress space as the full $C^{\text {div }}-\mathcal{P}_{k+1}$ space

$$
\Sigma_{h, k+1}:=\left\{\boldsymbol{\tau} \in H(\operatorname{div}, \Omega ; \mathbb{S})|\boldsymbol{\tau}|_{K} \in \mathcal{P}_{k+1}(K ; \mathbb{S}) \quad \forall K \in \mathcal{T}_{h}\right\},
$$

and take the discrete displacement space as the full $C^{-1}-\mathcal{P}_{k}$ space

$$
V_{h, k}:=\left\{v \in L^{2}\left(\Omega ; \mathbb{R}^{n}\right)|v|_{K} \in \mathcal{P}_{k}\left(K, \mathbb{R}^{n}\right) \quad \forall K \in \mathcal{T}_{h}\right\} .
$$

Then, the mixed finite element approximation of the elastic problem $(2.3)$ reads: Find $\left(\boldsymbol{\sigma}_{h}, u_{h}\right) \in \Sigma_{h, k+1} \times V_{h, k}$ such that

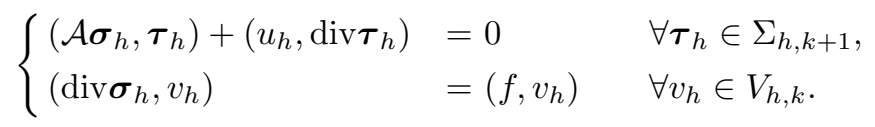

2.2. Stability and Convergence. The convergence of the finite element solution follows from the stability and the standard approximation property. First, we consider the stability of the discrete problem (2.6), which follows from two conditions by the standard theory of mixed finite element methods (cf. [18]).

(1) K-ellipticity: There exists a constant $\alpha>0$, independent of the grid size, such that

$$
\left(\mathcal{A} \boldsymbol{\tau}_{h}, \boldsymbol{\tau}_{h}\right) \geq \alpha\left\|\boldsymbol{\tau}_{h}\right\|_{H(\text { div })}^{2} \quad \forall \boldsymbol{\tau}_{h} \in Z_{h},
$$

where $Z_{h}:=\left\{\boldsymbol{\tau}_{h} \in \Sigma_{h, k+1} \mid\left(\operatorname{div} \boldsymbol{\tau}_{h}, v_{h}\right)=0 \quad \forall v_{h} \in V_{h, k}\right\}=\left\{\boldsymbol{\tau}_{h} \in\right.$ $\left.\Sigma_{h, k+1} \mid \operatorname{div} \boldsymbol{\tau}_{h}=0\right\}$. 
(2) Ladyženskaja-Babuška-Brezzi (LBB) condition: There exists a constant $\beta>0$, independent of the grid size, such that

$$
\inf _{v_{h} \in V_{h}} \sup _{\boldsymbol{\tau}_{h} \in \Sigma_{h, k+1}} \frac{\left(\operatorname{div} \boldsymbol{\tau}_{h}, v_{h}\right)}{\left\|\boldsymbol{\tau}_{h}\right\|_{H(\operatorname{div})}\left\|v_{h}\right\|_{0}} \geq \beta .
$$

Since $\operatorname{div} \Sigma_{h, k+1} \subset V_{h, k}$ for any $k \geq 0$, we know that $Z_{h} \in \operatorname{ker}(\operatorname{div})$. Therefore,

$$
\left(\mathcal{A} \boldsymbol{\tau}_{h}, \boldsymbol{\tau}_{h}\right) \geq C\left\|\boldsymbol{\tau}_{h}\right\|_{0}^{2}=C\left\|\boldsymbol{\tau}_{h}\right\|_{H(\text { div })}^{2} \quad \forall \boldsymbol{\tau}_{h} \in Z_{h},
$$

as the compliance tensor is positive definite. This implies the K-ellipticity. Note that, pertaining to $\int_{\Omega} \operatorname{tr}\left(\boldsymbol{\tau}_{h}\right) \mathrm{d} x=0$, the constant $C$ in 2.9$)$ is uniform with respect to the Poisson's' ratio $\left(\tilde{\nu}:=\frac{\tilde{\lambda}}{2(\tilde{\lambda}+\tilde{\mu})}\right)$ due to the following theorem (see Section 9 in 18. for details).

Theorem 2.1. Assume that $\boldsymbol{\sigma} \in H(\operatorname{div}, \Omega ; \mathbb{S})$ satisfies $\int_{\Omega} \operatorname{tr}(\boldsymbol{\sigma})=0$ and $\operatorname{div} \boldsymbol{\sigma}=0$. It holds that

$$
\|\boldsymbol{\sigma}\|_{0}^{2} \lesssim 2 \tilde{\mu}(\mathcal{A} \boldsymbol{\sigma}, \boldsymbol{\sigma}) .
$$

Next, we discuss the inf-sup condition under the pure displacement boundary condition. Similar techniques work for the traction boundary condition.

Lemma 2.2. When $k \geq n$, for any $v_{h} \in V_{h, k}$, there exists $\tau_{h} \in \Sigma_{h, k+1}$ such that

$$
\operatorname{div} \boldsymbol{\tau}_{h}=v_{h} \quad \text { and } \quad\left\|\boldsymbol{\tau}_{h}\right\|_{H(\text { div })} \lesssim\left\|v_{h}\right\|_{0} .
$$

Proof. This is a corollary of [32, 35, 36, in which a family of finite elements for $H(\operatorname{div}, \Omega ; \mathbb{S})$ satisfying $(2.11)$ is proposed as

$$
\begin{aligned}
\Sigma_{h, k+1}^{\mathrm{HZ}}:=\left\{\boldsymbol{\tau} \in H(\operatorname{div}, \Omega ; \mathbb{S}) \mid \boldsymbol{\tau}=\boldsymbol{\tau}_{c}+\boldsymbol{\tau}_{b}, \boldsymbol{\tau}_{c} \in H^{1}(\Omega ; \mathbb{S}),\right. & \\
& \left.\left.\boldsymbol{\tau}_{c}\right|_{K} \in \mathcal{P}_{k+1}(K ; \mathbb{S}),\left.\boldsymbol{\tau}_{b}\right|_{K} \in \Sigma_{k+1, b}(K) \quad \forall K \in \mathcal{T}_{h}\right\} .
\end{aligned}
$$

Here, the local conforming div-bubble space $\Sigma_{k+1, b}(K):=\left\{\boldsymbol{\tau} \in \mathcal{P}_{k+1}(K ; \mathbb{S})|\boldsymbol{\tau} \nu|_{\partial K}=\right.$ $0\}$. Hence, the lemma follows from the fact that $\boldsymbol{\tau}_{h} \in \Sigma_{h, k+1}^{\mathrm{HZ}} \subset \Sigma_{h, k+1}$.

For the lower order case, the inf-sup condition 2.11 resorts to some known results of the Stokes pair. When $k \geq n-2$, the Stokes pair $\mathcal{P}_{k+2}-\mathcal{P}_{k+1}^{-1}$ can be proved stable on special grids (cf. [10, 57]), a popular example of which is the Hsieh-Clough-Tocher (HCT) grid, where each macro-simplex is divided into $n+1$ sub-simplexes by connecting the barycenter with the vertices.

Lemma 2.3. When $n-2 \leq k<n$, if the Stokes pair $\mathcal{P}_{k+2}-\mathcal{P}_{k+1}^{-1}$ is stable on the grid, then for any $v_{h} \in V_{h, k}$, there exists $\boldsymbol{\tau}_{h} \in \Sigma_{h, k+1}$ such that

$$
\operatorname{div} \boldsymbol{\tau}_{h}=v_{h} \quad \text { and } \quad\left\|\boldsymbol{\tau}_{h}\right\|_{H(\operatorname{div})} \lesssim\left\|v_{h}\right\|_{0} .
$$

Proof. We prove the stability by a constructive method (cf. [8]). In light of the Brezzi-Douglas-Marini (BDM) elements for $H\left(\operatorname{div} ; \mathbb{R}^{n}\right)$ (cf. [17, 18]), we defined the following space

$$
\operatorname{BDM}_{k+1}^{n \times n}:=\left\{\boldsymbol{\tau} \in H(\operatorname{div}, \Omega ; \mathbb{M})|\boldsymbol{\tau}|_{K} \in \mathcal{P}_{k+1}(K ; \mathbb{M}) \quad \forall K \in \mathcal{T}_{h}\right\},
$$

where $\mathbb{M}$ represents the space of real matrices of order $n \times n$. The $\operatorname{div} \boldsymbol{\tau}$ here is defined by taking div on each row of $\tau$. By the stability of BDM elements, we immediately know that for any $v_{h} \in V_{h}$, there exists a $\tilde{\boldsymbol{\tau}}_{h} \in \mathrm{BDM}_{k+1}^{n \times n}$ such that

$$
\operatorname{div} \tilde{\boldsymbol{\tau}}_{h}=v_{h} \quad \text { and } \quad\left\|\tilde{\boldsymbol{\tau}}_{h}\right\|_{H(\text { div })} \lesssim\left\|v_{h}\right\|_{0} .
$$


With the purpose of symmetrizing $\boldsymbol{\tau}_{h}$, we add a divergence-free term to $\tilde{\boldsymbol{\tau}}_{h}$ to obtain

$$
\tau_{h}=\tilde{\boldsymbol{\tau}}_{h}+\operatorname{curl} \rho_{h},
$$

where $\rho_{h}$ satisfies

(1) For $n=2: \quad \rho_{h} \in H^{1}\left(\Omega ; \mathbb{R}^{2}\right)$ is a vector-valued function and $\left.\rho_{h}\right|_{K} \in$ $\mathcal{P}_{k+2}\left(K ; \mathbb{R}^{2}\right)$

(2) For $n=3: \quad \rho_{h} \in H^{1}(\Omega ; \mathbb{M})$ is a matrix-valued function and $\left.\rho_{h}\right|_{K} \in$ $\mathcal{P}_{k+2}(K ; \mathbb{M})$.

For the $2 \mathrm{D}$ case, the curl operator is a rotation of the operator $\nabla$ (i.e., curl $=$ $\left.\left(-\partial_{y}, \partial_{x}\right)\right)$ and applies on each entry of the vector $\rho_{h}$. For the 3D case, the curl operator applies on each row of the matrix $\rho_{h}$. By direct calculation, the symmetry of $\boldsymbol{\tau}_{h}$ is equivalent to the following equation,

$$
\operatorname{skw}\left(\operatorname{curl} \rho_{h}\right)=-\operatorname{skw} \tilde{\boldsymbol{\tau}}_{h},
$$

where $\operatorname{skw} \boldsymbol{\tau}:=\left(\boldsymbol{\tau}-\boldsymbol{\tau}^{T}\right) / 2$. For a scalar function $v$ and a vector-valued function $v=\left(v_{1}, v_{2}, v_{3}\right)^{T}$, we further define

$$
\operatorname{Skw}_{2}(v):=\left[\begin{array}{cc}
0 & v \\
-v & 0
\end{array}\right] \text { and } \operatorname{Skw}_{3}(v):=\left[\begin{array}{ccc}
0 & v_{3} & -v_{2} \\
-v_{3} & 0 & v_{1} \\
v_{2} & -v_{1} & 0
\end{array}\right] .
$$

Then, the proof can be divided into the following two cases:

(1) For $n=2$ : From [9], we have $\operatorname{skw}\left(\operatorname{curl} \rho_{h}\right)=\frac{1}{2} \operatorname{Skw}_{2}\left(\operatorname{div} \rho_{h}\right)$. Thus, 2.14 can be written as:

$$
\operatorname{div} \rho_{h}=\tilde{\tau}_{h, 21}-\tilde{\tau}_{h, 12} .
$$

The stability of Stokes pair $\mathcal{P}_{k+2}-\mathcal{P}_{k+1}^{-1}$ then implies that there exists a $\rho_{h} \in\left\{v \in H^{1}\left(\Omega ; \mathbb{R}^{2}\right)|v|_{K} \in \mathcal{P}_{k+2}\left(K ; \mathbb{R}^{2}\right)\right\}$ satisfying 2.15 and

$$
\left\|\rho_{h}\right\|_{1} \lesssim\left\|\tilde{\tau}_{h, 21}-\tilde{\tau}_{h, 12}\right\|_{0} \leq\left\|\tilde{\boldsymbol{\tau}}_{h}\right\|_{0} \lesssim\left\|v_{h}\right\|_{0} .
$$

(2) For $n=3$ : From [9], we have $\operatorname{skw}\left(\operatorname{curl} \rho_{h}\right)=-\frac{1}{2} \operatorname{Skw}_{3}\left(\operatorname{div} \Xi \rho_{h}\right)$, where $\Xi$ is an algebraic operator defined as $\Xi \rho_{h}=\rho_{h}^{T}-\operatorname{tr}\left(\rho_{h}\right) \boldsymbol{I}$. Denoting $\eta_{h}=\Xi \rho_{h}$, it is obvious that $\rho_{h}=\Xi^{-1} \eta_{h}=\eta_{h}^{T}-\frac{1}{2} \operatorname{tr}\left(\eta_{h}\right) \boldsymbol{I}$. Thus, 2.14 can be written as:

$$
\operatorname{div} \eta_{h}=\left(\tilde{\tau}_{h, 23}-\tilde{\tau}_{h, 32}, \tilde{\tau}_{h, 31}-\tilde{\tau}_{h, 13}, \tilde{\tau}_{h, 12}-\tilde{\tau}_{h, 21}\right)^{T} .
$$

Again, there exists a $\eta_{h} \in\left\{\boldsymbol{\tau} \in H^{1}(\Omega ; \mathbb{M})|\boldsymbol{\tau}|_{K} \in \mathcal{P}_{k+2}(K ; \mathbb{M})\right\}$ satisfying 2.16) and

$$
\left\|\rho_{h}\right\|_{1} \lesssim\left\|\eta_{h}\right\|_{1} \lesssim\left\|\tilde{\boldsymbol{\tau}}_{h}\right\|_{0} \lesssim\left\|v_{h}\right\|_{0}
$$

To summarize, we obtain $\boldsymbol{\tau}_{h}=\tilde{\boldsymbol{\tau}}_{h}+\operatorname{curl} \rho_{h}$ that satisfying $\boldsymbol{\tau}_{h} \in \Sigma_{h, k+1}$,

$$
\operatorname{div} \boldsymbol{\tau}_{h}=v_{h} \quad \text { and } \quad\left\|\boldsymbol{\tau}_{h}\right\|_{H(\text { div })} \lesssim\left\|\tilde{\boldsymbol{\tau}}_{h}\right\|_{H(\text { div })}+\left\|\operatorname{curl} \rho_{h}\right\|_{0} \lesssim\left\|v_{h}\right\|_{0} .
$$

This completes the proof.

By virtue of Lemma 2.2 and 2.3 , we have the following theorems.

Theorem 2.4. Under the conditions in Lemma 2.2 or 2.3, the K-ellipticity 2.7) and the inf-sup condition (2.8) hold uniformly with respect to the mesh size. Consequently, the discrete mixed problem (2.6) is well posed. 
Theorem 2.5. Let $(\boldsymbol{\sigma}, u) \in \Sigma \times V$ be the exact solution of the problem 2.3 and $\left(\boldsymbol{\sigma}_{h}, u_{h}\right) \in \Sigma_{h, k+1} \times V_{h, k}$ the finite element solution of 2.6). Assume that $\boldsymbol{\sigma} \in H^{k+2}(\Omega ; \mathbb{S})$ and $u \in H^{k+1}\left(\Omega ; \mathbb{R}^{n}\right)$. Under the conditions in Lemma 2.2 or 2.3. we have

$$
\left\|\boldsymbol{\sigma}-\boldsymbol{\sigma}_{h}\right\|_{H(\mathrm{div})}+\left\|u-u_{h}\right\|_{0} \lesssim h^{k+1}\left(|\boldsymbol{\sigma}|_{k+2}+|u|_{k+1}\right) .
$$

Proof. The well-posedness implies the following quasi-optimal error estimate,

$$
\left\|\boldsymbol{\sigma}-\boldsymbol{\sigma}_{h}\right\|_{H(\mathrm{div})}+\left\|u-u_{h}\right\|_{0} \lesssim \inf _{\boldsymbol{\tau}_{h} \in \Sigma_{h, k+1}, \boldsymbol{v}_{h} \in V_{h, k}}\left(\left\|\boldsymbol{\sigma}-\boldsymbol{\tau}_{h}\right\|_{H(\mathrm{div})}+\left\|u-v_{h}\right\|_{0}\right),
$$

which gives rise to (2.17) due to the standard $L^{2}$ projection and Scott-Zhang interpolation (cf. [51).

\section{HyBRIDIZATION}

To implement the mixed method (2.6), we need the degrees of freedom (d.o.f.) or the nodal basis of the discrete stress spaces. In the definition (2.4), however, we state the inter-element continuity directly instead of using the d.o.f., which is different from Ciarlet's convention for the finite elements. More precisely, there is no locally defined d.o.f. on elements for the discrete stress spaces 2.4. A similar argument can be found in [11]. In light of [4], where the authors constructed the nodal basis for the space of piecewise $C^{1}$ polynomials, we can globally form the nodal basis for our discrete stress spaces, whose dimensions depend on the singular vertices of the grids.

Instead of presenting the details of the nodal basis, we adopt a simpler implementation technique - the hybridization method (cf. [6, 24]), which imposes the interelement continuity by Lagrange multiplier. The hybridization method removes the inter-element continuity from the space $\Sigma_{h, k+1}$, which results in a discontinuous stress space

$$
\Sigma_{h, k+1}^{-1}:=\left\{\boldsymbol{\tau}_{h} \in L^{2}(\Omega ; \mathbb{S})\left|\boldsymbol{\tau}_{h}\right|_{K} \in \mathcal{P}_{k+1}(K ; \mathbb{S}) \quad \forall K \in \mathcal{T}_{h}\right\}
$$

To enforce the inter-element continuity of the stress, we introduce the Lagrange multiplier space $M_{h, k+1}$, where

$$
M_{h, k+1}:=\left\{\mu_{h} \in L^{2}\left(\mathcal{F}_{h}, \mathbb{R}^{n}\right)\left|\mu_{h}\right|_{F} \in \mathcal{P}_{k+1}\left(F, \mathbb{R}^{n}\right) \quad \forall F \in \mathcal{F}_{h}^{i} \text {, and }\left.\mu_{h}\right|_{\mathcal{F}_{h}^{\partial}}=0\right\} .
$$

The hybridized mixed finite element method is to find $\left(\boldsymbol{\sigma}_{h}, u_{h}, \lambda_{h}\right) \in \Sigma_{h, k+1}^{-1} \times$ $V_{h, k} \times M_{h, k+1}$ satisfying

$$
\begin{aligned}
\left(\mathcal{A} \boldsymbol{\sigma}_{h}, \boldsymbol{\tau}_{h}\right)_{\mathcal{T}_{h}}+\left(\operatorname{div}_{h} \boldsymbol{\tau}_{h}, u_{h}\right)_{\mathcal{T}_{h}}-\left\langle\left[\boldsymbol{\tau}_{h}\right], \lambda_{h}\right\rangle_{\mathcal{F}_{h}^{i}} & =0 & & \forall \boldsymbol{\tau}_{h} \in \Sigma_{h, k+1}^{-1} \\
\left(\operatorname{div}_{h} \boldsymbol{\sigma}_{h}, v_{h}\right)_{\mathcal{T}_{h}} & =\left(f, v_{h}\right) & & \forall v_{h} \in V_{h, k} \\
-\left\langle\left[\boldsymbol{\sigma}_{h}\right], \mu_{h}\right\rangle_{\mathcal{F}_{h}^{i}} & =0 & & \forall \mu_{h} \in M_{h, k+1}
\end{aligned}
$$

Here, $\operatorname{div}_{h}$ is the broken divergence operator. For convenience, let $\mathcal{B}:=\operatorname{div}_{h}$ : $\Sigma_{h, k+1}^{-1} \mapsto V_{h, k}$ and $\mathcal{C}: \Sigma_{h, k+1}^{-1} \mapsto M_{h, k+1}$ defined by

$$
\left.\mathcal{C} \boldsymbol{\tau}\right|_{F}:= \begin{cases}{\left.[\boldsymbol{\tau}]\right|_{F}} & \text { for } F \in \mathcal{F}_{h}^{i}, \\ 0 & \text { for } F \in \mathcal{F}_{h}^{\partial} .\end{cases}
$$


The adjoint of these operators are defined as $\mathcal{B}^{*}: V_{h, k} \mapsto \Sigma_{h, k+1}^{-1}$ and $\mathcal{C}^{*}: M_{h, k+1} \mapsto$ $\Sigma_{h, k+1}^{-1}$ such that for any $\left(\boldsymbol{\tau}_{h}, v_{h}, \mu_{h}\right) \in \Sigma_{h, k+1}^{-1} \times V_{h, k} \times M_{h, k+1}$,

$$
\left(\mathcal{B}^{*} v_{h}, \boldsymbol{\tau}_{h}\right)=\left(v_{h}, \mathcal{B} \boldsymbol{\tau}_{h}\right) \quad \text { and } \quad\left(\mathcal{C}^{*} \mu_{h}, \boldsymbol{\tau}_{h}\right)=\left\langle\mu_{h}, \mathcal{C} \boldsymbol{\tau}_{h}\right\rangle_{\mathcal{F}_{h}^{i}} .
$$

The following theorem shows the property of hybridized method given in 3.3 .

Theorem 3.1. There exists a solution $\left(\boldsymbol{\sigma}_{h}, u_{h}, \lambda_{h}\right) \in \Sigma_{h, k+1}^{-1} \times V_{h, k} \times M_{h, k+1}$ for the hybridized system (3.3). Moreover, the first two components of the solution are unique and coincide with that of the mixed method 2.6.

Proof. By Theorem 2.4 , there exists a solution $\left(\boldsymbol{\sigma}_{h}, u_{h}\right) \in \Sigma_{h, k+1} \times V_{h, k}$ for the mixed method 2.6). It is obvious that $\left(\boldsymbol{\sigma}_{h}, u_{h}\right)$ satisfies the last two equations (3.3b) and 3.3c). The first equation 3.3a) can be written as

$$
\mathcal{C}^{*} \lambda_{h}=\mathcal{A} \sigma_{h}+\mathcal{B}^{*} u_{h}
$$

Since $\mathrm{R}\left(\mathcal{C}^{*}\right)^{\perp}=\operatorname{ker}(\mathcal{C})$ and $\operatorname{ker}(\mathcal{C})=\Sigma_{h, k+1}$, we have

$$
\mathrm{R}\left(\mathcal{C}^{*}\right)=\left(\Sigma_{h, k+1}\right)^{\perp} .
$$

Here, $\left(\Sigma_{h, k+1}\right)^{\perp}$ is the $L^{2}$ orthogonal complement of $\Sigma_{h, k+1}$ in $\Sigma_{h, k+1}^{-1}$ with respect to the inner product $(\cdot, \cdot)$. Since $\left(\boldsymbol{\sigma}_{h}, u_{h}\right)$ satisfies (3.3a) for $\boldsymbol{\tau}_{h} \in \Sigma_{h, k+1}$, it holds that

$$
\mathcal{A} \boldsymbol{\sigma}_{h}+\mathcal{B}^{*} u_{h} \in\left(\Sigma_{h, k+1}\right)^{\perp} .
$$

Hence, there exists $\lambda_{h} \in M_{h, k+1}$ satisfying (3.5), which indicates the existence of the solution for 3.3 .

For the uniqueness, assuming that $\left(\boldsymbol{\sigma}_{h}, u_{h}, \lambda_{h}\right)$ satisfies (3.3), then (3.3c) implies that $\boldsymbol{\sigma}_{h} \in \Sigma_{h, k+1}$. Moreover, since $\Sigma_{h, k+1} \subset \Sigma_{h, k+1}^{-1}$, choosing $\boldsymbol{\tau}_{h} \in \Sigma_{h, k+1}$, we can see that the system (3.3a) and (3.3b) is identical to the system of the mixed method (2.6). Therefore, $\left(\boldsymbol{\sigma}_{h}, u_{h}\right)$ solves (2.6). The uniqueness of $\left(\boldsymbol{\sigma}_{h}, u_{h}\right)$ follows from Theorem 2.4. This completes the proof.

Remark 3.2. We note that Hu-Zhang elements in 2.12 can also be written as

$$
\begin{aligned}
\Sigma_{h, k+1}^{\mathrm{HZ}}=\{\boldsymbol{\tau} \in H(\operatorname{div}, \Omega ; \mathbb{S}) \mid & \left.\boldsymbol{\tau}\right|_{K} \in \mathcal{P}_{k+1}(K ; \mathbb{S}) \quad \forall K \in \mathcal{T}_{h}, \\
& \text { and } \left.\left.\boldsymbol{\tau}\right|_{a} \text { is continuous for any } a \in \mathcal{N}_{h}\right\} .
\end{aligned}
$$

We enrich the space $\Sigma_{h, k+1}^{\mathrm{HZ}}$ by relaxing the continuity on the element vertices. Similar technique can be used in Arnold-Winther [1] $(n=2)$ or Arnold-AwanouWinther [5] $(n=3)$ elements $\tilde{\Sigma}_{h, k+n-}-V_{h, k}$, where

$$
\begin{aligned}
\tilde{\Sigma}_{h, k+n-}:=\{\boldsymbol{\tau} \in H(\operatorname{div}, \Omega ; \mathbb{S}) \mid & \left.\boldsymbol{\tau}\right|_{K} \in \tilde{\Sigma}_{k+n-}(K) \quad \forall K \in \mathcal{T}_{h}, \\
& \text { and } \left.\left.\boldsymbol{\tau}\right|_{a} \text { is continuous for any } a \in \mathcal{N}_{h}\right\} .
\end{aligned}
$$

and $\tilde{\Sigma}_{k+n-}(K):=\left\{\boldsymbol{\tau} \in \mathcal{P}_{k+n}(K ; \mathbb{S}) \mid \operatorname{div} \boldsymbol{\tau} \in \mathcal{P}_{k}\left(K ; \mathbb{R}^{n}\right)\right\}$. We denote the hybridized version of $\tilde{\Sigma}_{h, k+n-}$ by

$$
\Sigma_{h, k+n-}:=\left\{\boldsymbol{\tau} \in H(\operatorname{div}, \Omega ; \mathbb{S})|\boldsymbol{\tau}|_{K} \in \tilde{\Sigma}_{k+n-}(K) \quad \forall K \in \mathcal{T}_{h}\right\} .
$$

Table 1 compares $\tilde{\Sigma}_{h, k+n-}$ and $\Sigma_{h, k+1}^{\mathrm{HZ}}$ to their hybridized versions. 
TABLE 1. $\tilde{\Sigma}_{h, k+n-}, \Sigma_{h, k+1}^{\mathrm{HZ}}$ and their hybridized versions.

\begin{tabular}{|c|c|c|c|c|}
\hline Elements & Gerneral grids & Special grids & Hybridizable & Lagrange multiplier \\
\hline$\Sigma_{h, k+n-}-V_{h, k}$ & $k \geq 1$ & - & $\times$ & - \\
$\Sigma_{h, k+1}^{\mathrm{HZ}}-V_{h, k}$ & $k \geq n$ & - & $\times$ & - \\
$\Sigma_{h, k+n-}-V_{h, k}$ & $k \geq 1$ & - & $\sqrt{ }$ & $M_{h, k+n}$ \\
$\Sigma_{h, k+1}-V_{h, k}$ & $k \geq n$ & $k \geq n-2$ & $\sqrt{ }$ & $M_{h, k+1}$ \\
\hline
\end{tabular}

3.1. Kernel of the Hybrid System. Theorem 3.1 implies that the kernel of the hybridized mixed system $(3.3)$ is $\{0\} \times\{0\} \times \operatorname{ker}\left(\mathcal{C}^{*}\right)$. It is straightforward that $\operatorname{ker}\left(\mathcal{C}^{*}\right)=\mathrm{R}(\mathcal{C})^{\perp}$, where $\mathrm{R}(\mathcal{C})^{\perp}$ is the $L^{2}$ orthogonal complement of $\mathrm{R}(\mathcal{C})$ in the space $M_{h, k+1}$ with respect to the inner product $\langle\cdot, \cdot\rangle_{\mathcal{F}_{h}^{i}}$. We therefore have the following decomposition for the multiplier space

$$
M_{h, k+1}=\mathrm{R}(\mathcal{C}) \oplus \mathrm{R}(\mathcal{C})^{\perp} .
$$

We note that the dimension of $\mathrm{R}(\mathcal{C})^{\perp}$ depends on the grid.

Definition 3.3 (44) $)$. In the $2 \mathrm{D}$ case, an interior vertex $a \in \mathcal{N}_{h}(a \notin \partial \Omega)$ is called singular, if and only if the edges meeting at this vertex fall on two straight lines.

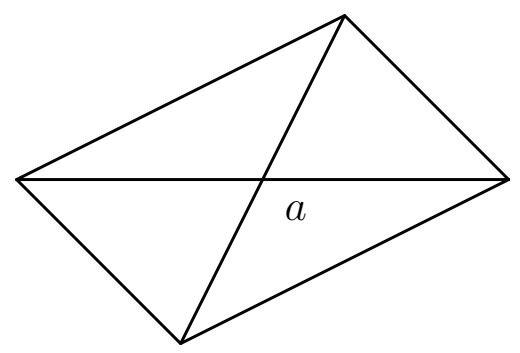

FIGURE 1. Singular vertex $a$.

Lemma 3.4. Both $\mathrm{R}(\mathcal{C})$ and $\mathrm{R}(\mathcal{C})^{\perp}$ have local basis, that is,

$$
\begin{aligned}
\mathrm{R}(\mathcal{C}) & =\operatorname{span}\left\{\varphi_{1}, \varphi_{2}, \cdots, \varphi_{N_{1}}\right\}, \\
\mathrm{R}(\mathcal{C})^{\perp} & =\operatorname{span}\left\{\psi_{1}, \psi_{2}, \cdots, \psi_{N_{2}}\right\},
\end{aligned}
$$

where $\varphi_{i}, \psi_{j} \in M_{h, k+1}$ are locally supported and $N_{1}, N_{2}$ are the dimensions of the spaces $\mathrm{R}(\mathcal{C}), \mathrm{R}(\mathcal{C})^{\perp}$, respectively. Moreover, for the $2 D$ case, if there is no interior singular vertex in $\mathcal{T}_{h}$, we have $N_{2}=0$ and $\left\{\varphi_{1}, \cdots, \varphi_{N_{1}}\right\}$ can be chosen such that the mass matrix $\boldsymbol{M}=\left(\left\langle\varphi_{i}, \varphi_{j}\right\rangle_{\mathcal{F}_{h}^{i}}\right)$ is well-conditioned, that is,

$$
\sum_{i=1}^{N_{1}} c_{i}^{2}\left\|\varphi_{i}\right\|_{0}^{2} \approx\left\|\sum_{i=1}^{N_{1}} c_{i} \varphi_{i}\right\|_{0}^{2} \quad \forall\left(c_{1}, c_{2}, \cdots, c_{N_{1}}\right) \in \mathbb{R}^{N_{1}} .
$$

Proof. The detailed proof is given in the appendix. 
3.2. SPSD System for Lagrange Multiplier. In this subsection, we eliminate the variable $\boldsymbol{\sigma}_{h}$ and $u_{h}$ in the hybridized mixed system (3.3), then obtain a linear system solely for $\lambda_{h}$.

For any $\lambda \in M_{h, k+1}$, we define two local problems:

(1) Find $\left(\boldsymbol{\sigma}_{\lambda}, u_{\lambda}\right) \in \Sigma_{h, k+1}^{-1} \times V_{h, k}$ such that for any element $K \in \mathcal{T}_{h}$,

$$
\begin{aligned}
\left(\mathcal{A} \boldsymbol{\sigma}_{\lambda}, \boldsymbol{\tau}_{h}\right)_{K}+\left(u_{\lambda}, \operatorname{div} \boldsymbol{\tau}_{h}\right)_{K} & =\left\langle\lambda, \boldsymbol{\tau}_{h} \nu\right\rangle_{\partial K} & & \forall \boldsymbol{\tau}_{h} \in \mathcal{P}_{k+1}(K ; \mathbb{S}), \\
\left(\operatorname{div} \boldsymbol{\sigma}_{\lambda}, v_{h}\right)_{K} & =0 & & \forall v_{h} \in \mathcal{P}_{k}\left(K ; \mathbb{R}^{n}\right) .
\end{aligned}
$$

(2) Find $\left(\tilde{\boldsymbol{\sigma}}_{f}, \tilde{u}_{f}\right) \in \Sigma_{h, k+1}^{-1} \times V_{h, k}$ such that for any element $K \in \mathcal{T}_{h}$,

$$
\begin{aligned}
\left(\mathcal{A} \tilde{\boldsymbol{\sigma}}_{f}, \boldsymbol{\tau}_{h}\right)_{K}+\left(\tilde{u}_{f}, \operatorname{div} \boldsymbol{\tau}_{h}\right)_{K} & =0 & & \forall \boldsymbol{\tau}_{h} \in \mathcal{P}_{k+1}(K ; \mathbb{S}), \\
\left(\operatorname{div} \tilde{\boldsymbol{\sigma}}_{f}, v_{h}\right)_{K} & =\left(f, v_{h}\right)_{K} & & \forall v_{h} \in \mathcal{P}_{k}\left(K ; \mathbb{R}^{n}\right) .
\end{aligned}
$$

The following lemma shows that both $\left(\boldsymbol{\sigma}_{m}, u_{m}\right)$ and $\left(\tilde{\boldsymbol{\sigma}}_{f}, \tilde{u}_{f}\right)$ are well defined.

Lemma 3.5. The systems (3.8) and (3.9) are unisolvent. Moreover, the solution of the system (3.3) satisfies

$$
\boldsymbol{\sigma}_{h}=\boldsymbol{\sigma}_{\lambda_{h}}+\tilde{\boldsymbol{\sigma}}_{f} \quad \text { and } \quad u_{h}=u_{\lambda_{h}}+\tilde{u}_{f} .
$$

Proof. The proof is similar to the standard one given in [24] and is therefore omitted here.

Note that $\left(\boldsymbol{\sigma}_{\lambda_{h}}, u_{\lambda_{h}}\right)$ and $\left(\tilde{\boldsymbol{\sigma}}_{f}, \tilde{u}_{f}\right)$ can be computed element by element. The above lemma means that the $\boldsymbol{\sigma}_{h}$ and $u_{h}$ can be locally recovered after solving the variable $\lambda_{h}$.

Theorem 3.6. The Lagrange multiplier $\lambda_{h}$ satisfies

$$
s\left(\lambda_{h}, \mu_{h}\right)=-\left(f, u_{\mu_{h}}\right) \quad \forall \mu \in M_{h, k+1},
$$

where $s\left(\lambda_{h}, \mu_{h}\right)=\left(\mathcal{A} \boldsymbol{\sigma}_{\lambda_{h}}, \boldsymbol{\sigma}_{\mu_{h}}\right)$. Moreover, the system (3.11) is symmetric positivesemidefinite and its kernel is $\mathrm{R}(\mathcal{C})^{\perp}$.

Proof. The derivation of (3.11) is standard in the hybridization method (cf. 24]). The kernel of the multiplier system is the same with the hybridized mixed system.

3.3. Norm Estimates. We denote the linear operator corresponding to the bilinear form $s(\cdot, \cdot)$ by $\mathcal{S}: M_{h, k+1} \mapsto M_{h, k+1}$, or $S: M_{h, k+1} \mapsto M_{h, k+1}^{\prime}$ as

$$
\langle S \lambda, \mu\rangle:=\langle\mathcal{S} \lambda, \mu\rangle_{\mathcal{F}_{h}^{i}}:=s(\lambda, \mu) \quad \forall \lambda, \mu \in M_{h, k+1} .
$$

In fact, $\mathcal{S}$ is the Schur complement of the hybridized mixed system (3.3). In light of Theorem 3.6, we can define a norm $\|\cdot\|_{S}$ on $\mathrm{R}(\mathcal{C})$ as

$$
\|\lambda\|_{S}^{2}:=\sum_{K \in \mathcal{T}_{h}}\|\lambda\|_{S, K}^{2}:=\sum_{K \in \mathcal{T}_{h}}\left(\mathcal{A} \boldsymbol{\sigma}_{\lambda}, \boldsymbol{\sigma}_{\lambda}\right)_{K} \quad \forall \lambda \in \mathrm{R}(\mathcal{C}),
$$

which can also be extended as a semi-norm on $M_{h, k+1}$. For the conciseness, we still denote the semi-norm on $M_{h, k+1}$ by $\|\cdot\|_{S}$. 
To investigate how $\|\cdot\|_{S}$ depends on the parameters, we define the following semi-norms locally:

$$
\begin{aligned}
|\lambda|_{h, K}:=\sup _{\boldsymbol{\tau} \in Z_{h}(K)} \frac{\langle\lambda, \boldsymbol{\tau} \nu\rangle_{\partial K}}{\|\boldsymbol{\tau}\|_{0, K}} & \forall \lambda \in M_{h, k+1}, \\
|\lambda|_{*, K}:=|K|^{-1 / 2}\left|\int_{\partial K} \lambda \cdot \nu d s\right| & \forall \lambda \in M_{h, k+1} .
\end{aligned}
$$

Here, $Z_{h}(K)=\left\{\boldsymbol{\tau}_{h} \in \mathcal{P}_{k+1}(K ; \mathbb{S}) \mid \operatorname{div} \boldsymbol{\tau}_{h}=0\right\}$. The semi-norms $|\cdot|_{h}$ and $|\cdot|_{*}$ on $M_{h, k+1}$ are defined by the summations of local norms over all elements, namely,

$$
|\lambda|_{h}^{2}=\sum_{K \in \mathcal{T}_{h}}|\lambda|_{h, K}^{2} \quad \text { and } \quad|\lambda|_{*}^{2}=\sum_{K \in \mathcal{T}_{h}}|\lambda|_{*, K}^{2} .
$$

The relationship between $\|\cdot\|_{S}$ and $|\cdot|_{*},|\cdot|_{h}$ is described in the following lemma.

Theorem 3.7. It holds that

$$
\|\lambda\|_{S, K}^{2} \approx 2 \tilde{\mu}|\lambda|_{h, K}^{2}+\tilde{\lambda}|\lambda|_{*, K}^{2} \quad \forall \lambda \in M_{h, k+1} .
$$

Proof. Notice that $\left.\boldsymbol{\sigma}_{\lambda}\right|_{K} \in Z_{h}(K)$ by $(3.8 \mathrm{~b})$. Moreover, for any $\boldsymbol{\tau} \in Z_{h}(K)$, by (3.8a), we have

$$
\left(\mathcal{A} \sigma_{\lambda}, \tau\right)_{K}=\langle\lambda, \tau \nu\rangle_{\partial K}
$$

Let $m_{\boldsymbol{\tau}}=\frac{1}{n|K|} \int_{K} \operatorname{tr}(\boldsymbol{\tau}) d x$ and $\boldsymbol{\tau}_{0}=\boldsymbol{\tau}-m_{\boldsymbol{\tau}} \boldsymbol{I}$. Then $\left(\boldsymbol{\tau}_{0}, \boldsymbol{I}\right)_{K}=0$ and $\left(\mathcal{A} \boldsymbol{\tau}_{0}, \boldsymbol{I}\right)_{K}=$ 0 , which implies that

$$
(\mathcal{A} \boldsymbol{\tau}, \boldsymbol{\tau})_{K}=\left(\mathcal{A} \boldsymbol{\tau}_{0}, \boldsymbol{\tau}_{0}\right)_{K}+\left(\mathcal{A} m_{\boldsymbol{\tau}} \boldsymbol{I}, m_{\boldsymbol{\tau}} \boldsymbol{I}\right)_{K} .
$$

Let $\|\boldsymbol{\tau}\|_{\mathcal{A}, K}:=(\mathcal{A} \boldsymbol{\tau}, \boldsymbol{\tau})_{K}^{1 / 2}$ for any $\boldsymbol{\tau} \in L^{2}(K ; \mathbb{S})$. In light of 3.17 and 2.10 , we have for any $\lambda \in M_{h, k+1}$,

$$
\begin{aligned}
\|\lambda\|_{S, K} & =\sup _{\boldsymbol{\tau} \in Z_{h}(K)} \frac{\left(\mathcal{A} \boldsymbol{\sigma}_{\lambda}, \boldsymbol{\tau}\right)_{K}}{\|\boldsymbol{\tau}\|_{\mathcal{A}, K}}=\sup _{\boldsymbol{\tau} \in Z_{h}(K)} \frac{\langle\lambda, \boldsymbol{\tau} \nu\rangle_{\partial K}}{\|\boldsymbol{\tau}\|_{\mathcal{A}, K}} \\
& \leq \sup _{\boldsymbol{\tau} \in Z_{h}(K)} \frac{\left\langle\lambda, \boldsymbol{\tau}_{0} \nu\right\rangle_{\partial K}}{\|\boldsymbol{\tau}\|_{\mathcal{A}, K}}+\sup _{\boldsymbol{\tau} \in Z_{h}(K)} \frac{\left\langle\lambda, m_{\boldsymbol{\tau}} \boldsymbol{I} \nu\right\rangle_{\partial K}}{\|\boldsymbol{\tau}\|_{\mathcal{A}, K}} \\
& =\sup _{\boldsymbol{\tau} \in Z_{h}(K)} \frac{\left\langle\lambda, \boldsymbol{\tau}_{0} \nu\right\rangle_{\partial K}}{\left\|\boldsymbol{\tau}_{0}\right\|_{\mathcal{A}, K}}+\sup _{\boldsymbol{\tau} \in Z_{h}(K)} \frac{\left\langle\lambda, m_{\boldsymbol{\tau}} \boldsymbol{I} \nu\right\rangle_{\partial K}}{\left\|m_{\boldsymbol{\tau}} \boldsymbol{I}\right\|_{\mathcal{A}, K}} \\
& \lesssim(2 \tilde{\mu})^{1 / 2} \sup _{\boldsymbol{\tau} \in Z_{h}(K)} \frac{\left\langle\lambda, \boldsymbol{\tau}_{0} \nu\right\rangle_{\partial K}}{\left\|\boldsymbol{\tau}_{0}\right\|_{0, K}}+\tilde{\lambda}^{1 / 2}|\lambda|_{*, K} \\
& \lesssim(2 \tilde{\mu})^{1 / 2}|\lambda|_{h, K}+\tilde{\lambda}^{1 / 2}|\lambda|_{*, K} .
\end{aligned}
$$

On the other hand, since $2 \tilde{\mu}(\mathcal{A} \boldsymbol{\tau}, \boldsymbol{\tau})_{K} \lesssim\|\boldsymbol{\tau}\|_{0, K}^{2}$ by the definition of $\mathcal{A}$, we have

$$
(2 \tilde{\mu})^{1 / 2}|\lambda|_{h, K}=(2 \tilde{\mu})^{1 / 2} \sup _{\boldsymbol{\tau} \in Z_{h}(K)} \frac{\langle\lambda, \boldsymbol{\tau} \nu\rangle_{\partial K}}{\|\boldsymbol{\tau}\|_{0, K}} \lesssim \sup _{\boldsymbol{\tau} \in Z_{h}(K)} \frac{\langle\lambda, \boldsymbol{\tau} \nu\rangle_{\partial K}}{\|\boldsymbol{\tau}\|_{\mathcal{A}, K}}=\|\lambda\|_{S, K} .
$$

Moreover, we have $\left(\mathcal{A} \boldsymbol{\sigma}_{\lambda}, \boldsymbol{I}\right)_{K}=\langle\lambda, \boldsymbol{I} \nu\rangle_{\partial K}$ from 3.8a. By the Cauchy-Schwarz inequality, we have

$$
\begin{aligned}
\tilde{\lambda}^{1 / 2}|\lambda|_{*, K} & =\tilde{\lambda}^{1 / 2}|K|^{-1 / 2}\left|\langle\lambda, \boldsymbol{I} \nu\rangle_{\partial K}\right|=\tilde{\lambda}^{1 / 2}|K|^{-1 / 2}\left|\left(\mathcal{A} \boldsymbol{\sigma}_{\lambda}, \boldsymbol{I}\right)_{K}\right| \\
& \leq \tilde{\lambda}^{1 / 2}|K|^{-1 / 2}\|\lambda\|_{S, K}(\mathcal{A} \boldsymbol{I}, \boldsymbol{I})_{K}^{1 / 2} \leq\|\lambda\|_{S, K} .
\end{aligned}
$$

This completes the proof. 
Next, we estimate the condition number of $S$. The $L^{2}$ norm for $M_{h, k+1}$ is denoted by

$$
\|\lambda\|_{0}^{2}:=\sum_{F \in \mathcal{F}_{h}^{i}}\|\lambda\|_{0, F}^{2}:=\sum_{F \in \mathcal{F}_{h}^{i}}\langle\lambda, \lambda\rangle_{F} .
$$

Lemma 3.8. It holds that

$$
\|\lambda\|_{S}^{2} \lesssim(2 \tilde{\mu}+\tilde{\lambda}) h^{-1}\|\lambda\|_{0}^{2} \quad \forall \lambda \in M_{h, k+1} .
$$

Proof. The upper bound of $S$ follows from the equivalent norm 3.16), CauchySchwarz inequality and standard scaling argument.

The lower bound of $S$ depends on the singularity of the grids. In light of [50], we define a quantity to measure the vertex singularity. The rest estimates are focused on the case of spatial dimension $n=2$. For a vertex $a \in \mathcal{N}_{h}$, let $\theta_{i}, 1 \leq i \leq m$ be the angles of the triangle $K_{i}$ meeting at $a$ (triangles are numbered consecutively). If $a$ is an internal vertex, we define

$$
\kappa(a):=\max \left\{\left|\theta_{i}+\theta_{j}-\pi\right| \mid 1 \leq i, j \leq m \text { and } i-j=1 \quad \bmod m\right\} ;
$$

If $a$ is a boundary vertex, $\kappa(a)$ is defined in the same way without the modulo operation. We further set

$$
\kappa=\min _{a \in \mathcal{N}_{h}} \kappa(a) .
$$

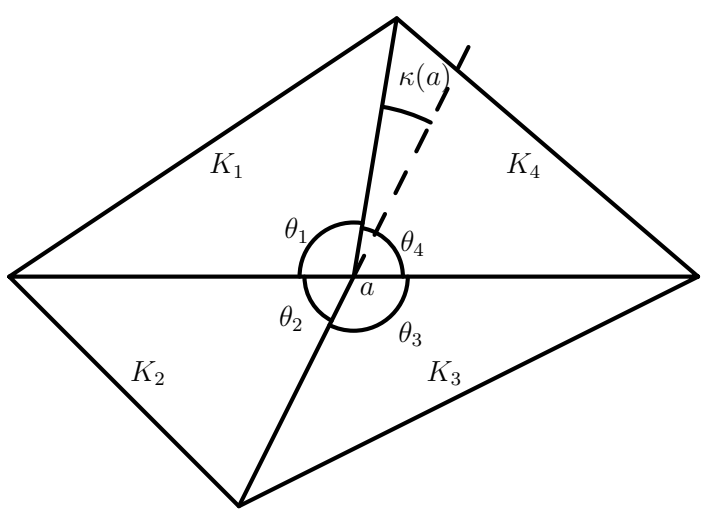

FiguRE 2. Nearly singular vertex.

In the following, we assume that $\kappa \geq \kappa_{0}>0$, where $\kappa_{0}$ is a positive constant independent of $h$. That is, there is no singular or nearly singular vertex on $\mathcal{T}_{h}$.

Lemma 3.9. Assume that $\kappa \geq \kappa_{0}>0$. For any local basis function $\varphi_{i}$ of $\mathrm{R}(\mathcal{C})$ (see Lemma 3.4), there exists a locally supported $\boldsymbol{\tau}_{i} \in \Sigma_{h, k+1}^{-1}$ such that

$$
\left.\left[\boldsymbol{\tau}_{i}\right]\right|_{F}=\left.\varphi_{i}\right|_{F} \quad \forall F \in \mathcal{F}_{h}, \quad \text { and } \quad\left\|\boldsymbol{\tau}_{i}\right\|_{0}^{2} \lesssim \frac{h}{\sin ^{2}\left(\kappa_{0}\right)}\left\|\varphi_{i}\right\|_{0}^{2} .
$$

Proof. The detail proof for above lemma will be given in the appendix.

Lemma 3.10. Assume that $\kappa \geq \kappa_{0}>0$. For any $\lambda \in M_{h, k+1}$, there exists $\boldsymbol{\tau} \in$ $\Sigma_{h, k+1}^{-1}$ such that

$$
\left.[\boldsymbol{\tau}]\right|_{F}=\left.\lambda\right|_{F} \quad \forall F \in \mathcal{F}_{h}, \quad \text { and } \quad\|\boldsymbol{\tau}\|_{0}^{2} \lesssim \frac{h}{\sin ^{2}\left(\kappa_{0}\right)}\|\lambda\|_{0}^{2} .
$$


Proof. Since $\kappa \geq \kappa_{0}>0$, by Lemma 3.4 we have $M_{h, k+1}=\mathrm{R}(\mathcal{C})$ and there exists a local basis that satisfies (3.7). Therefore, any $\lambda \in M_{h, k+1}$ can be uniquely expressed as

$$
\lambda=\sum_{i=1}^{N_{1}} c_{i} \varphi_{i} \quad \text { and } \quad \sum_{i=1}^{N_{1}}\left\|c_{i} \varphi_{i}\right\|_{0}^{2} \approx\|\lambda\|_{0}^{2} .
$$

By virtue of Lemma 3.9 , there exists a locally supported $\boldsymbol{\tau}_{i} \in \Sigma_{h, k+1}^{-1}$ for each basis function $\varphi_{i}$ of $\mathrm{R}(\mathcal{C})$, such that

$$
\left.\left[\boldsymbol{\tau}_{i}\right]\right|_{F}=\left.\varphi_{i}\right|_{F} \quad \forall F \in \mathcal{F}_{h}, \quad \text { and } \quad\left\|\boldsymbol{\tau}_{i}\right\|_{0}^{2} \lesssim h \sin ^{-2}\left(\kappa_{0}\right)\left\|\varphi_{i}\right\|_{0}^{2} .
$$

Therefore, $\boldsymbol{\tau}=\sum_{i=1}^{N_{1}} c_{i} \boldsymbol{\tau}_{i}$ satisfies $\left.[\boldsymbol{\tau}]\right|_{F}=\lambda$ and

$$
\|\boldsymbol{\tau}\|_{0}^{2} \lesssim \sum_{i=1}^{N_{1}} c_{i}^{2}\left\|\boldsymbol{\tau}_{i}\right\|_{0}^{2} \lesssim h \sin ^{-2}\left(\kappa_{0}\right) \sum_{i=1}^{N_{1}} c_{i}^{2}\left\|\varphi_{i}\right\|_{0}^{2} \approx h \sin ^{-2}\left(\kappa_{0}\right)\|\lambda\|_{0}^{2} .
$$

This completes the proof.

Lemma 3.11. Assume that $\kappa \geq \kappa_{0}>0$. It holds that

$$
2 \tilde{\mu} h \sin ^{2}\left(\kappa_{0}\right)\|\lambda\|_{0}^{2} \lesssim\|\lambda\|_{S}^{2} \quad \forall \lambda \in M_{h, k+1} .
$$

Proof. By virtue of Lemma 3.10 for any $\lambda \in M_{h, k+1}$, there exists $\boldsymbol{\tau}_{1} \in \Sigma_{h, k+1}^{-1}$ such that

$$
\mathcal{C} \boldsymbol{\tau}_{1}=\lambda \quad \text { and } \quad\left\|\boldsymbol{\tau}_{1}\right\|_{0} \lesssim h^{1 / 2} \sin ^{-1}\left(\kappa_{0}\right)\|\lambda\|_{0}
$$

Applying the discrete inf-sup condition, there exists $\boldsymbol{\tau}_{2} \in \Sigma_{h, k+1}$ such that

$\operatorname{div} \boldsymbol{\tau}_{2}=-\operatorname{div}_{h} \boldsymbol{\tau}_{1} \quad$ and $\quad\left\|\boldsymbol{\tau}_{2}\right\|_{H(\operatorname{div})} \lesssim\left\|\operatorname{div}_{h} \boldsymbol{\tau}_{1}\right\|_{0} \lesssim h^{-1}\left\|\boldsymbol{\tau}_{1}\right\|_{0} \lesssim h^{-1 / 2} \sin ^{-1}\left(\kappa_{0}\right)\|\lambda\|_{0}$.

Let $\boldsymbol{\tau}=\boldsymbol{\tau}_{1}+\boldsymbol{\tau}_{2}$. Thus, $\operatorname{div}_{h} \boldsymbol{\tau}=0$ and $\mathcal{C} \boldsymbol{\tau}=\lambda$. By summation of 3.8a) over all elements and choosing above $\boldsymbol{\tau}$ as a testing function, we have

$$
\|\lambda\|_{0}^{2}=\left(\mathcal{A} \boldsymbol{\sigma}_{\lambda}, \boldsymbol{\tau}\right) \leq\left(\mathcal{A} \boldsymbol{\sigma}_{\lambda}, \boldsymbol{\sigma}_{\lambda}\right)^{1 / 2}(2 \tilde{\mu})^{-1 / 2}\|\boldsymbol{\tau}\|_{0} \lesssim(2 \tilde{\mu} h)^{-1 / 2} \sin ^{-1}\left(\kappa_{0}\right)\|\lambda\|_{S}\|\lambda\|_{0},
$$

which implies 3.20 .

Lemmas 3.8 and 3.11 imply the following condition number estimate:

$$
\operatorname{cond}(S) \lesssim \frac{2 \tilde{\mu}+\tilde{\lambda}}{2 \tilde{\mu}} h^{-2} \sin ^{-2}\left(\kappa_{0}\right) .
$$

For the nearly incompressible material, $\tilde{\lambda}$ would be sufficient large, which makes the multiplier system (3.11) nearly singular.

\section{Multilevel Solvers for the Hybridized Mixed Methods}

In this section, we shall describe several multilevel solvers for the hybridized mixed methods for the $2 \mathrm{D}$ case. We further assume that $\kappa \geq \kappa_{0}>0$, which is guaranteed when the grid has no singular or nearly singular vertex. 
4.1. Two-level and multilevel solvers. First, we present the two-level solvers. We consider an overlapping decomposition $\left\{\Omega_{i}\right\}_{i=1}^{J}$, where $\Omega_{i}$ are open subdomains of $\Omega$. Let $\mathcal{T}_{H}$ be a coarse grid for $\Omega$, and $\mathcal{T}_{h}$ be a subdivision of $\mathcal{T}_{H}$ such that $\mathcal{T}_{h}$ is aligned with each $\partial \Omega_{i}$. We assume that there exist nonnegative $C^{\infty}$ functions $\theta_{1}, \theta_{2}, \cdots, \theta_{J}$ in $\mathbb{R}^{2}$ such that

$$
\begin{aligned}
& \theta_{i}=0 \quad \text { on } \Omega \backslash \Omega_{i}, \\
& \sum_{i=1}^{J} \theta_{i}=1 \quad \text { on } \bar{\Omega}, \\
& \left\|\nabla \theta_{i}\right\|_{\infty} \lesssim \delta^{-1} .
\end{aligned}
$$

Here, $\delta>0$ is a parameter that measures the overlap among the subdomains. We also assume that there exists an integer $N_{c}$ independent of $h, \delta$, and $J$ such that any point in $\Omega$ belongs to at most $N_{c}$ subdomains. The local space associated with subdomain $\Omega_{i}$ is denoted by

$$
M_{i}:=\left\{\lambda \in M_{h, k+1}|\lambda|_{F}=0 \text {, for any face } F \in \Omega \backslash \Omega_{i}\right\} .
$$

We can then define $S_{i}: M_{i} \mapsto M_{i}^{\prime}$ and bilinear form on $M_{i}$ by

$$
\left\langle S_{i} \lambda_{i}, \mu_{i}\right\rangle:=s_{i}\left(\lambda_{i}, \mu_{i}\right):=s\left(\iota_{i} \lambda_{i}, \iota_{i} \mu_{i}\right),
$$

where $\iota_{i}: M_{i} \hookrightarrow M_{h, k+1}$ denotes the inclusion operator.

In light of the multigrid method on the primal elasticity problem by Schöberl 48, we choose the continuous and piecewise quadratic finite element space as the coarse space

$$
W_{H}:=\left\{w \in H_{0}^{1}\left(\Omega ; \mathbb{R}^{2}\right)|w|_{K} \in \mathcal{P}_{2}\left(K ; \mathbb{R}^{2}\right) \quad \forall K \in \mathcal{T}_{H}\right\}
$$

Suppose the coarse space $W_{H}$ is connected to $M_{h, k+1}$ by an injective intergrid operator $I_{H}^{h}: W_{H} \mapsto M_{h, k+1}$ (The construction of $I_{H}^{h}$ will be given in Section 4.2). We will impose the primal elastic norm $\|\cdot\|_{A_{H}}$ on $W_{H}$, where $A_{H}: W_{H} \mapsto W_{H}^{\prime}$ and the bilinear form $a_{H}(\cdot, \cdot)$ are defined as

$$
\begin{aligned}
\left\langle A_{H} w_{H}, v_{H}\right\rangle & :=a_{H}\left(w_{H}, v_{H}\right) \\
& :=2 \tilde{\mu}\left(\boldsymbol{\epsilon}\left(w_{H}\right), \boldsymbol{\epsilon}\left(v_{H}\right)\right)+\tilde{\lambda}\left(P_{0}^{H} \operatorname{div} w_{H}, P_{0}^{H} \operatorname{div} v_{H}\right) \quad \forall w_{H}, v_{H} \in W_{H}, \\
\left\|w_{H}\right\|_{A_{H}}^{2} & :=a_{H}\left(w_{H}, w_{H}\right) \quad \forall w_{H} \in W_{H} .
\end{aligned}
$$

Here, $P_{0}^{H}$ is the $L^{2}$ projection on the piecewise constant space on $\mathcal{T}_{H}$. Then, the two-level additive Schwarz preconditioner can be constructed as

$$
B_{\mathrm{ad}}=I_{H}^{h} A_{H}^{-1}\left(I_{H}^{h}\right)^{\prime}+\sum_{i=1}^{J} \iota_{i} S_{i}^{-1} \iota_{i}^{\prime} .
$$

Our main contribution is the following estimate.

Theorem 4.1. The condition number of $B_{\mathrm{ad}} S$ satisfies

$$
\operatorname{cond}\left(B_{\mathrm{ad}} S\right) \leq C\left(1+N_{c}\right) \frac{H^{2}}{\delta^{2}},
$$

where $C$ is independent to both the mesh size $h$ and the Lamé constants. 
According to [54, 53], the estimate of the condition number of the additive Schwarz method is based on the stability of the intergrid transfer operator $I_{H}^{h}$ (Lemma 4.4) and the stable decomposition (Theorem 4.9), which will be proved in the rest of this section.

Now, we are ready to introduce the multilevel preconditioner as follows:

$$
\tilde{B}_{\mathrm{ad}}=I_{H}^{h} B_{H}\left(I_{H}^{h}\right)^{\prime}+\sum_{i=1}^{J} \iota_{i} S_{i}^{-1} \iota_{i}^{\prime} .
$$

Here, $B_{H}: W_{H}^{\prime} \mapsto W_{H}$ is the multilevel preconditioner for $A_{H}$, see [48, 49. Then we have the following theorem.

Theorem 4.2. If

$$
\left\langle B_{H}^{-1} w_{H}, w_{H}\right\rangle \approx\left\langle A_{H} w_{H}, w_{H}\right\rangle \quad \forall w_{H} \in W_{H},
$$

then the condition number of $\tilde{B}_{\text {ad }} S$ satisfies

$$
\operatorname{cond}\left(\tilde{B}_{\mathrm{ad}} S\right) \leq C\left(1+N_{c}\right) \frac{H^{2}}{\delta^{2}},
$$

where $C$ is independent to the mesh size $h$ and the Lamé constants.

Proof. It follows directly from Theorem 4.1 and norm equivalence between $\|\cdot\|_{A_{H}}$ and $\|\cdot\|_{B_{H}^{-1}}$.

Remark 4.3. (1) When $H \lesssim \delta$, the preconditioners (4.4) and 4.5) are both uniform with respect to $h$ and the Lamé constants.

(2) It can be proved that the corresponding multiplicative preconditioners are also uniform as well as the additive version, provided that the local problems associated with the subdomains are solved exactly (cf. [21, 38, 3]).

(3) Some robust multilevel methods to solve the linear elasticity problem can be found in [16, 40, 25, 31, 20. By constructing stable intergrid transfer operators similar to $I_{H}^{h}$, it is feasible to construct corresponding multilevel solvers to the hybridized mixed method.

4.2. Intergrid Transfer Operator $I_{H}^{h}$. The construction of intergrid transfer operator $I_{H}^{h}$ is divided into two steps: (i) the intergrid transfer operator from coarse grid to fine grid proposed by Schöberl [48, and (ii) the $L^{2}$ projection operator to Lagrange multiplier space. More precisely, we first define the $\mathcal{P}_{2}$ Lagrange finite element space $W_{h}$ on $\mathcal{T}_{h}$

$$
W_{h}:=\left\{w \in H_{0}^{1}\left(\Omega ; \mathbb{R}^{2}\right)|w|_{K} \in \mathcal{P}_{2}\left(K ; \mathbb{R}^{2}\right) \quad \forall K \in \mathcal{T}_{h}\right\},
$$

with the primal elastic norm $\|\cdot\|_{A_{h}}$, bilinear form $a_{h}(\cdot, \cdot)$, and $A_{h}: W_{h} \mapsto W_{h}^{\prime}$ similar to 4.3. In [48, the harmonic extension $\tilde{I}_{H}^{h}: W_{H} \mapsto W_{h}$ was defined as follows: For $w_{H} \in W_{H}$, the value of $\tilde{I}_{H}^{h} w_{H}$ on each edge of coarse element $K_{H} \in \mathcal{T}_{H}$ does not change, and the value in the interior of $K_{H}$ is defined by discrete harmonic extension, that is,

$$
\begin{aligned}
\left.\tilde{I}_{H}^{h} w_{H}\right|_{\partial K_{H}} & =\left.w_{H}\right|_{\partial K_{H}}, \\
a_{h}\left(\tilde{I}_{H}^{h} w_{H}, v_{h}\right) & =0 \quad \forall v_{h} \in W_{h, 0}\left(K_{H}\right),
\end{aligned}
$$

where $W_{h, 0}\left(K_{H}\right):=\left\{w \in H_{0}^{1}\left(K_{H} ; \mathbb{R}^{2}\right)|w|_{K^{\prime}} \in \mathcal{P}_{2}\left(K^{\prime} ; \mathbb{R}^{2}\right) \quad \forall K^{\prime} \in K_{H}\right\} . \tilde{I}_{H}^{h}$ has the following stability property (cf. [48]),

$$
\left\|\tilde{I}_{H}^{h} w_{H}\right\|_{A_{h}} \lesssim\left\|w_{H}\right\|_{A_{H}} \quad \forall w_{H} \in W_{H} .
$$


The intergrid transfer operator $I_{H}^{h}$ appearing in 4.4 is defined as the product of two operators,

$$
I_{H}^{h}:=Q_{h} \tilde{I}_{H}^{h}: W_{H} \mapsto M_{h, k+1},
$$

where $Q_{h}: W_{h} \mapsto M_{h, k+1}$ is the $L^{2}$ projection on edges (i.e., $\left\langle Q_{h} w_{h}, \mu\right\rangle_{\mathcal{F}_{h}}:=$ $\left.\left\langle w_{h}, \mu\right\rangle_{\mathcal{F}_{h}}, \forall \mu \in M_{h, k+1}\right)$. Then, we have the following lemma:

Lemma 4.4. The intergrid transfer operator $I_{H}^{h}: W_{H} \mapsto M_{h, k+1}$ has the following stability property:

$$
\left\|I_{H}^{h} w_{H}\right\|_{S} \lesssim\left\|w_{H}\right\|_{A_{H}} \quad \forall w_{H} \in W_{H} .
$$

Proof. Note that $Q_{h}$ is the $L^{2}$ projection on $M_{h, k+1}$. Then, for any $w_{h} \in W_{h}$,

$$
\begin{aligned}
\left|Q_{h} w_{h}\right|_{*, K} & =|K|^{-1 / 2}\left|\int_{\partial K} Q_{h} w_{h} \cdot \nu d s\right|=|K|^{-1 / 2}\left|\int_{\partial K} w_{h} \cdot \nu d s\right| \\
& =|K|^{-1 / 2}\left|\int_{K} \operatorname{div} w_{h} d x\right|=\left\|P_{0}^{h} \operatorname{div} w_{h}\right\|_{0, K},
\end{aligned}
$$

and

$$
\begin{aligned}
\left|Q_{h} w_{h}\right|_{h, K} & =\sup _{\boldsymbol{\tau} \in Z_{h}(K)} \frac{\left\langle Q_{h} w_{h}, \boldsymbol{\tau} \nu\right\rangle_{\partial K}}{\|\boldsymbol{\tau}\|_{0, K}}=\sup _{\boldsymbol{\tau} \in Z_{h}(K)} \frac{\left\langle w_{h}, \boldsymbol{\tau} \nu\right\rangle_{\partial K}}{\|\boldsymbol{\tau}\|_{0, K}} \\
& =\sup _{\boldsymbol{\tau} \in Z_{h}(K)} \frac{\left(\epsilon\left(w_{h}\right), \boldsymbol{\tau}\right)_{K}}{\|\boldsymbol{\tau}\|_{0, K}} \leq\left\|\epsilon\left(w_{h}\right)\right\|_{0, K},
\end{aligned}
$$

which implies that $\left\|Q_{h} w_{h}\right\|_{S} \lesssim\left\|w_{h}\right\|_{A_{h}}$ due to Theorem 3.7. The stability property 4.9 then follows from (4.7) and the stability property of $Q_{h}$.

4.3. Stable Decomposition. In this section, we shall present the stable decomposition. A key tool to prove the stable decomposition is the interpolation $\Pi_{h}$ : $M_{h, k+1} \mapsto W_{h}$, which is used to capture the low-frequency of the multiplier $\lambda \in$ $M_{h, k+1}$.

We first define a parameter-independent problem: Find $\left(\overline{\boldsymbol{\sigma}}_{\lambda}, \bar{u}_{\lambda}\right) \in \Sigma_{h, k+1}^{-1} \times V_{h, k}$ such that for any element $K \in \mathcal{T}_{h}$,

$$
\begin{array}{lll}
\left(\overline{\boldsymbol{\sigma}}_{\lambda}, \boldsymbol{\tau}_{h}\right)_{K}+\left(\bar{u}_{\lambda}, \operatorname{div} \boldsymbol{\tau}_{h}\right)_{K}=\left\langle\lambda, \boldsymbol{\tau}_{h} \nu\right\rangle_{\partial K} & & \forall \boldsymbol{\tau}_{h} \in \mathcal{P}_{k+1}(K ; \mathbb{S}), \\
\left(\operatorname{div} \overline{\boldsymbol{\sigma}}_{\lambda}, v_{h}\right)_{K} & =0 & \forall v_{h} \in \mathcal{P}_{k}\left(K ; \mathbb{R}^{2}\right) .
\end{array}
$$

We then introduce the following rigid motion space on each element $K$,

$$
\operatorname{RM}(K):=\left\{v \in H^{1}\left(K, \mathbb{R}^{2}\right) \mid\left(\nabla v+(\nabla v)^{T}\right) / 2=0\right\} .
$$

We also introduce a projection $P_{K, \mathrm{RM}}: M_{h, k+1}(\partial K) \mapsto \mathrm{RM}(K)$ by

$$
\left(P_{K, \mathrm{RM}} \lambda, r\right)_{K}=\left(\bar{u}_{\lambda}, r\right)_{K} \quad \forall r \in \operatorname{RM}(K) .
$$

Then, the construction of the interpolation $\Pi_{h}$ is divided into two steps. First, a Clément type interpolation $\Pi_{1, h}: M_{h, k+1} \mapsto\left(\mathcal{P}_{1, h}\right)^{2} \cap H^{1}\left(\Omega ; \mathbb{R}^{2}\right)$ is defined as, for any $a \in \mathcal{N}_{h}$,

$$
\left(\Pi_{1, h} \lambda\right)(a):=\left\{\begin{array}{cc}
\frac{\sum_{K \in \omega_{a}}\left(P_{K, \mathrm{RM}} \lambda\right)(a)}{\sum_{K \in \omega_{a}} 1} & a \notin \partial \Omega, \\
0 & a \in \partial \Omega,
\end{array}\right.
$$


where $\mathcal{P}_{1, h}$ is the piecewise linear Lagrange element and $\omega_{a}$ is the set of elements containing the vertex $a$. Next, we define the correction operator $\Pi_{2, h}: M_{h, k+1} \cup$ $H_{1}\left(\Omega, \mathbb{R}^{2}\right) \mapsto W_{h}:$

$$
\left(\Pi_{2, h} \lambda\right)(a):=0 \quad \forall a \in \mathcal{N}_{h}, \quad \text { and } \quad \int_{F} \Pi_{2, h} \lambda d s:=\int_{F} \lambda d s \quad \forall F \in \mathcal{F}_{h} .
$$

Then, the interpolation $\Pi_{h}$ is composed by these two operators,

$$
\Pi_{h} \lambda:=\Pi_{1, h} \lambda+\Pi_{2, h}\left(\lambda-\Pi_{1, h} \lambda\right) \quad \forall \lambda \in M_{h, k+1} .
$$

Note that the interpolation $\Pi_{h}$ is only used for analysis and will not occur in the computation. To prove the stability and approximation property of $\Pi_{h}$, we present some lemmas on $P_{K, \mathrm{RM}}$.

Lemma 4.5. It holds that

$$
h_{K}^{-1}\left\|\bar{u}_{\lambda}-P_{K, \mathrm{RM}} \lambda\right\|_{0, K} \lesssim|\lambda|_{h, K} .
$$

Proof. By definition, we have $\left(\bar{u}_{\lambda}-P_{K, \mathrm{RM}} \lambda\right) \in \mathrm{RM}(K)^{\perp}$. According to the Theorem 2.2 in 32 , we can find $\tilde{\boldsymbol{\tau}}_{h} \in \Sigma_{k+1, b}(K)$ such that

$$
\operatorname{div} \tilde{\boldsymbol{\tau}}_{h}=\bar{u}_{\lambda}-P_{K, \mathrm{RM}} \lambda \quad \text { and } \quad h_{K}^{-1}\left\|\tilde{\boldsymbol{\tau}}_{h}\right\|_{0, K} \lesssim\left\|\operatorname{div} \tilde{\boldsymbol{\tau}}_{h}\right\|_{0}=\left\|\bar{u}_{\lambda}-P_{K, \mathrm{RM}} \lambda\right\|_{0, K} .
$$

Since $\left.\tilde{\boldsymbol{\tau}}_{h} \nu\right|_{\partial K}=0,4.10 \mathrm{a}$ implies that

$$
\left(\overline{\boldsymbol{\sigma}}_{\lambda}, \tilde{\boldsymbol{\tau}}_{h}\right)_{K}+\left(\bar{u}_{\lambda}-P_{K, \mathrm{RM}} \lambda, \operatorname{div} \tilde{\boldsymbol{\tau}}_{h}\right)_{K}=0 .
$$

Thus,

$$
\begin{aligned}
\left\|\bar{u}_{\lambda}-P_{K, \mathrm{RM}} \lambda\right\|_{0, K}^{2} & =\left(\bar{u}_{\lambda}-P_{K, \mathrm{RM}} \lambda, \operatorname{div} \tilde{\boldsymbol{\tau}}_{h}\right)_{K}=-\left(\overline{\boldsymbol{\sigma}}_{\lambda}, \tilde{\boldsymbol{\tau}}_{h}\right) \leq\left\|\overline{\boldsymbol{\sigma}}_{\lambda}\right\|_{0, K}\left\|\tilde{\boldsymbol{\tau}}_{h}\right\|_{0, K} \\
& \lesssim h_{K}\left\|\bar{u}_{\lambda}-P_{K, \mathrm{RM}} \lambda\right\|_{0, K}\left\|\overline{\boldsymbol{\sigma}}_{\lambda}\right\|_{0, K}
\end{aligned}
$$

Note that $\overline{\boldsymbol{\sigma}}_{\lambda} \in Z_{h}(K)$ by $4.10 \mathrm{~b}$. By definition of $|\cdot|_{h, K}$ in 3.14 , we have

$$
|\lambda|_{h, K}=\sup _{\boldsymbol{\tau}_{h} \in Z_{h}(K)} \frac{\left\langle\lambda, \boldsymbol{\tau}_{h} \nu\right\rangle_{\partial K}}{\left\|\boldsymbol{\tau}_{h}\right\|_{0, K}}=\sup _{\boldsymbol{\tau}_{h} \in Z_{h}(K)} \frac{\left(\overline{\boldsymbol{\sigma}}_{h}, \boldsymbol{\tau}_{h}\right)_{K}}{\left\|\boldsymbol{\tau}_{h}\right\|_{0, K}}=\left\|\overline{\boldsymbol{\sigma}}_{\lambda}\right\|_{0, K} .
$$

Then, 4.14 follows from the above two equations.

Lemma 4.6. Assume that $\kappa \geq \kappa_{0}>0$. It holds that

$$
h_{K}^{-1}\left\|\lambda-P_{K, \mathrm{RM}} \lambda\right\|_{0, \partial K}^{2} \lesssim \sum_{K^{\prime} \in \omega_{K}}|\lambda|_{h, K^{\prime}}^{2}
$$

Proof. By virtue of Lemma 4.5 the triangle inequality, and the trace inequality, we only need to prove

$$
h_{K}^{-1}\left\|\lambda-\bar{u}_{\lambda}\right\|_{0, \partial K}^{2} \lesssim \sum_{K^{\prime} \in \omega_{K}}|\lambda|_{h, K^{\prime}}^{2}
$$

Consider the element patch $\omega_{K}$. Let $\mathcal{F}_{h}\left(\omega_{K}\right):=\mathcal{F}_{h} \cap \bar{\omega}_{K}$ and $\Sigma_{h, k+1}^{-1}\left(\omega_{K}\right):=$ $\left\{\boldsymbol{\tau}_{h} \in L^{2}\left(\omega_{K} ; \mathbb{S}\right)\left|\boldsymbol{\tau}_{h}\right|_{K}^{\prime} \in \mathcal{P}_{k+1}\left(K^{\prime} ; \mathbb{S}\right) \quad \forall K^{\prime} \in \omega_{K}\right\}$. By summation of 4.10a over elements $K^{\prime} \in \omega_{K}$, we have

$$
\left(\overline{\boldsymbol{\sigma}}_{\lambda}, \boldsymbol{\tau}_{h}\right)_{\omega_{K}}+\left(\bar{u}_{\lambda}, \operatorname{div}_{h} \boldsymbol{\tau}_{h}\right)_{\omega_{K}}=\sum_{K^{\prime} \in \omega_{K}}\left\langle\lambda, \boldsymbol{\tau}_{h} \nu\right\rangle_{\partial K^{\prime}} \quad \forall \boldsymbol{\tau}_{h} \in \Sigma_{h, k+1}^{-1}\left(\omega_{K}\right) .
$$


Note that $\left.\bar{u}_{\lambda}\right|_{K} \in \mathcal{P}_{k}\left(K ; \mathbb{R}^{2}\right)$, we denote by $\bar{u}_{K}$ the natural continuous extension of $\left.\bar{u}_{\lambda}\right|_{K}$ on $\omega_{K}$ (i.e., $\bar{u}_{K}$ and $\left.\bar{u}_{\lambda}\right|_{K}$ have the same polynomial form). Then, we can recast 4.17) as

$$
\left(\overline{\boldsymbol{\sigma}}_{\lambda}, \boldsymbol{\tau}_{h}\right)_{\omega_{K}}+\left(\bar{u}_{\lambda}-\bar{u}_{K}, \operatorname{div}_{h} \boldsymbol{\tau}_{h}\right)_{\omega_{K}}-\left(\epsilon\left(\bar{u}_{K}\right), \boldsymbol{\tau}_{h}\right)_{\omega_{K}}=\sum_{F \in \mathcal{F}_{h}\left(\omega_{K}\right)}\left\langle\lambda-\bar{u}_{K},\left[\boldsymbol{\tau}_{h}\right]\right\rangle_{F} .
$$

Since $\kappa \geq \kappa_{0}>0$, by Lemma 3.10 there exists $\boldsymbol{\tau}_{1} \in \Sigma_{h, k+1}^{-1}\left(\omega_{K}\right)$ such that

$$
\left.\left[\boldsymbol{\tau}_{1}\right]\right|_{F}=\left\{\begin{array}{ll}
\left.\left(\lambda-u_{K}\right)\right|_{F} & F \in \partial K, \\
0 & \text { otherwise, }
\end{array} \quad \text { and } \quad\left\|\boldsymbol{\tau}_{1}\right\|_{0, \omega_{K}}^{2} \lesssim h_{K} \sin ^{-2}\left(\kappa_{0}\right)\left\|\lambda-u_{K}\right\|_{0, \partial K}^{2} .\right.
$$

Apply Lemma 2.2 or 2.3 on $\omega_{K}$, we immediately know that there exists $\tau_{2} \in$ $\Sigma_{h, k+1}\left(\omega_{K}\right):=\left\{\boldsymbol{\tau} \in H\left(\operatorname{div}, \omega_{K} ; \mathbb{S}\right)|\boldsymbol{\tau}|_{K^{\prime}} \in \mathcal{P}_{k+1}\left(K^{\prime} ; \mathbb{S}\right) \quad \forall K^{\prime} \in \omega_{K}\right\}$ such that

$\operatorname{div} \boldsymbol{\tau}_{2}=-\operatorname{div}_{h} \boldsymbol{\tau}_{1} \quad$ and $\quad h_{K}^{-1}\left\|\boldsymbol{\tau}_{2}\right\|_{0, \omega_{K}}+\left\|\operatorname{div} \boldsymbol{\tau}_{2}\right\|_{0, \omega_{K}} \lesssim\left\|\operatorname{div} \boldsymbol{\tau}_{1}\right\|_{0, \omega_{K}} \lesssim h_{K}^{-1}\left\|\boldsymbol{\tau}_{1}\right\|_{0, \omega_{K}}$.

Next, there is a unique decomposition that $\left.\bar{u}_{K}\right|_{K}=\theta_{1}+\theta_{2} \in \mathrm{RM}(K) \oplus \operatorname{RM}(K)^{\perp}$. Due to Theorem 2.2 in 32 , we can find $\tilde{\boldsymbol{\tau}}_{3} \in \Sigma_{k+1, b}(K)$ such that

$$
\operatorname{div} \tilde{\boldsymbol{\tau}}_{3}=\theta_{2} \quad \text { and } \quad h_{K}^{-1}\left\|\tilde{\boldsymbol{\tau}}_{3}\right\|_{0, K} \lesssim\left\|\theta_{2}\right\|_{0, K} .
$$

Let $\operatorname{supp}\left(\boldsymbol{\tau}_{3}\right) \subset K$ and

$$
\left.\boldsymbol{\tau}_{3}\right|_{K}= \begin{cases}\frac{\left(\boldsymbol{\tau}_{1}+\boldsymbol{\tau}_{2}, \epsilon\left(\bar{u}_{K}\right)\right)_{\omega_{K}}}{\left\|\theta_{2}\right\|_{0, K}^{2}} \tilde{\boldsymbol{\tau}}_{3} & \theta_{2} \neq 0 \\ \mathbf{0} & \theta_{2}=0\end{cases}
$$

A straightforward calculation shows that $\boldsymbol{\tau}_{3}$ satisfies

$$
-\left(\boldsymbol{\tau}_{3}, \epsilon\left(\bar{u}_{K}\right)\right)_{K}=\left(\operatorname{div} \boldsymbol{\tau}_{3}, \theta_{2}\right)_{K}=\left(\boldsymbol{\tau}_{1}+\boldsymbol{\tau}_{2}, \epsilon\left(\bar{u}_{K}\right)\right)_{\omega_{K}},
$$

and

$$
\begin{aligned}
\left\|\boldsymbol{\tau}_{3}\right\|_{0, K} & \leq\left\|\boldsymbol{\tau}_{1}+\boldsymbol{\tau}_{2}\right\|_{0, \omega_{K}} \frac{\left\|\epsilon\left(\bar{u}_{K}\right)\right\|_{0, \omega_{K}}\left\|\tilde{\boldsymbol{\tau}}_{3}\right\|_{0, K}}{\left\|\theta_{2}\right\|_{0, K}^{2}} \\
& \lesssim\left\|\boldsymbol{\tau}_{1}+\boldsymbol{\tau}_{2}\right\|_{0, \omega_{K}} \frac{h_{K}\left\|\epsilon\left(\bar{u}_{K}\right)\right\|_{0, K}}{\left\|\theta_{2}\right\|_{0, K}} \lesssim\left\|\boldsymbol{\tau}_{1}+\boldsymbol{\tau}_{2}\right\|_{0, \omega_{K}}
\end{aligned}
$$

Thus, take $\boldsymbol{\tau}=\boldsymbol{\tau}_{1}+\boldsymbol{\tau}_{2}+\boldsymbol{\tau}_{3}$ in 4.18, we have

$$
\left.[\boldsymbol{\tau}]\right|_{F}=\left\{\begin{array}{ll}
\left.\left(\lambda-u_{K}\right)\right|_{F} & F \in \partial K, \\
0 & \text { otherwise, }
\end{array}\left(\bar{u}_{\lambda}-\bar{u}_{K}, \operatorname{div} \boldsymbol{\tau}\right)_{\omega_{K}}=0 \quad \text { and } \quad\left(\epsilon\left(\bar{u}_{K}\right), \boldsymbol{\tau}\right)_{\omega_{K}}=0 .\right.
$$

In addition, 4.19, 4.20, and 4.22 imply that

$$
\|\boldsymbol{\tau}\|_{0, \omega_{K}} \lesssim\left\|\boldsymbol{\tau}_{1}\right\|_{0, \omega_{K}} \lesssim h_{K}^{1 / 2} \sin ^{-1}\left(\kappa_{0}\right)\left\|\lambda-\bar{u}_{K}\right\|_{0, \partial K} .
$$

Hence, we have

$$
\begin{aligned}
\left\|\lambda-\bar{u}_{K}\right\|_{0, \partial K}^{2} & =\left(\overline{\boldsymbol{\sigma}}_{\lambda}, \boldsymbol{\tau}\right)_{\omega_{K}} \lesssim\left\|\overline{\boldsymbol{\sigma}}_{\lambda}\right\|_{0, \omega_{K}}\|\boldsymbol{\tau}\|_{0, \omega_{K}} \\
& \lesssim\left(\sum_{K^{\prime} \in \omega_{K}}|\lambda|_{h, K^{\prime}}^{2}\right)^{1 / 2} h_{K}^{1 / 2} \sin ^{-1}\left(\kappa_{0}\right)\left\|\lambda-u_{K}\right\|_{0, \partial K},
\end{aligned}
$$

which gives rise to 4.16).

Now, we are in the place to prove the stability and approximation property of $\Pi_{h}$. 
Lemma 4.7. For any $\lambda \in M_{h, k+1}$, it holds that

$$
\begin{aligned}
\int_{F} \Pi_{h} \lambda d s & =\int_{F} \lambda d s \quad \forall F \in \mathcal{F}_{h}, \\
\left\|\Pi_{h} \lambda\right\|_{A_{h}} & \lesssim\|\lambda\|_{S}, \\
\left\|\lambda-Q_{h} \Pi_{h} \lambda\right\|_{0}^{2} & \lesssim h\|\lambda\|_{S}^{2} .
\end{aligned}
$$

Proof. By the definition of $\Pi_{h}$ in (4.13), we have

$$
\int_{F} \lambda-\Pi_{h} \lambda d s=\int_{F}\left(I-\Pi_{2, h}\right)\left(I-\Pi_{1, h}\right) \lambda d s=0,
$$

which gives rise to 4.23).

Since $\left\|\Pi_{h} \lambda\right\|_{A_{h}}^{2}=2 \tilde{\mu}\left\|\epsilon\left(\Pi_{h} \lambda\right)\right\|_{0}^{2}+\tilde{\lambda}\left\|P_{0}^{h} \operatorname{div}\left(\Pi_{h} \lambda\right)\right\|_{0}^{2}$, we prove the stability 4.24 of $\Pi_{h}$ part by part. By (4.23), we have

$$
\begin{aligned}
\left\|P_{0}^{h} \operatorname{div} \Pi_{h} \lambda\right\|_{0, K} & =|K|^{-1 / 2}\left|\int_{K} \operatorname{div}\left(\Pi_{h} \lambda\right) d x\right|=|K|^{-1 / 2}\left|\int_{\partial K}\left(\Pi_{h} \lambda\right) \cdot \nu d s\right| \\
& =|K|^{-1 / 2}\left|\int_{\partial K} \lambda \cdot \nu d s\right|=|\lambda|_{*, K} .
\end{aligned}
$$

Next, we estimate $\left\|\epsilon\left(\Pi_{h} \lambda\right)\right\|_{0, K}$. First, we show the stability of $\Pi_{1, h}$ as

$$
\begin{aligned}
\left\|\Pi_{1, h} \lambda-P_{K, \mathrm{RM}} \lambda\right\|_{0, K}^{2} & \lesssim h_{K}^{n} \sum_{a \in \mathcal{N}_{K}}\left|\left(\Pi_{1, h} \lambda\right)(a)-\left(P_{K, \mathrm{RM}} \lambda\right)(a)\right|^{2} \\
& \lesssim h_{K}^{n} \sum_{a \in \mathcal{N}_{K}} \sum_{\bar{K}_{1} \cap \bar{K}_{2}=\bar{F}}\left|\left(P_{K_{1}, \mathrm{RM}} \lambda\right)(a)-\left(P_{K_{2}, \mathrm{RM}} \lambda\right)(a)\right|^{2} \\
& \lesssim h_{K}^{n} \sum_{a \in \mathcal{N}_{K}} \sum_{\bar{K}_{1} \cap \bar{K}_{2}=\bar{F}}|\lambda|_{F}(a)-\left.\left(P_{K_{1}, \mathrm{RM}} \lambda\right)(a)\right|^{2}+|\lambda|_{F}(a)-\left.\left(P_{K_{2}, \mathrm{RM}} \lambda\right)(a)\right|^{2} \\
& \lesssim \sum_{a \in \mathcal{N}_{K}} \sum_{\bar{K}_{1} \cap \bar{K}_{2}=\bar{F}} h_{K_{1}}\left\|\lambda-P_{K_{1}, \mathrm{RM}} \lambda\right\|_{0, \partial K_{1}}^{2}+h_{K_{2}}\left\|\lambda-P_{K_{2}, \mathrm{RM}} \lambda\right\|_{0, \partial K_{2}}^{2} \\
& \lesssim \sum_{K^{\prime} \in \omega_{K}} h_{K^{\prime}}\left\|\lambda-P_{K^{\prime}, \mathrm{RM}} \lambda\right\|_{0, \partial K^{\prime}}^{2} .
\end{aligned}
$$

Then, by the triangle inequality and inverse inequality, we have

$$
\begin{aligned}
\left\|\Pi_{h} \lambda-P_{K, \mathrm{RM}} \lambda\right\|_{0, K}^{2} & \lesssim\left\|\Pi_{h} \lambda-\Pi_{1, h} \lambda\right\|_{0, K}^{2}+\left\|\Pi_{1, h} \lambda-P_{K, \mathrm{RM}} \lambda\right\|_{0, K}^{2} \\
& =\left\|\Pi_{2, h}\left(I-\Pi_{1, h}\right) \lambda\right\|_{0, K}^{2}+\left\|\Pi_{1, h} \lambda-P_{K, \mathrm{RM}} \lambda\right\|_{0, K}^{2} \\
& \lesssim h_{K}\left\|\left(I-\Pi_{1, h}\right) \lambda\right\|_{0, \partial K}^{2}+\left\|\Pi_{1, h} \lambda-P_{K, \mathrm{RM}} \lambda\right\|_{0, K}^{2} \\
& \lesssim \sum_{K^{\prime} \in \omega_{K}} h_{K^{\prime}}\left\|\lambda-P_{K^{\prime}, r m} \lambda\right\|_{0, \partial K^{\prime}}^{2} \quad \text { (by 4.27) }
\end{aligned}
$$

Hence, by the inverse inequality, we have

$$
\begin{aligned}
\left\|\epsilon\left(\Pi_{h} \lambda\right)\right\|_{0, K}^{2} & =\left\|\epsilon\left(\Pi_{h} \lambda-P_{K, \mathrm{RM}} \lambda\right)\right\|_{0, K}^{2} \\
& \lesssim h_{K}^{-2}\left\|\Pi_{h} \lambda-P_{K, \mathrm{RM}} \lambda\right\|_{0, K}^{2} \\
& \left.\lesssim \sum_{K^{\prime} \in \omega_{K}} h_{K^{\prime}}^{-1}\left\|\lambda-P_{K^{\prime}, \mathrm{RM}} \lambda\right\|_{0, \partial K^{\prime}}^{2} \quad \text { (by 4.28) }\right)
\end{aligned}
$$


By virtue of (4.15), we sum (4.26) and 4.29 over all elements to obtain

$$
\left\|\Pi_{h} \lambda\right\|_{A_{h}} \lesssim\|\lambda\|_{S}
$$

Next, the approximation property 4.25) can be obtained by summing the following inequalities over all elements:

$$
\begin{aligned}
\left\|\lambda-Q_{h} \Pi_{h} \lambda\right\|_{0, \partial K}^{2} & \lesssim\left\|\left(I-Q_{h}\right) \Pi_{h} \lambda\right\|_{0, \partial K}^{2}+\left\|\lambda-P_{K, \mathrm{RM}} \lambda\right\|_{0, \partial K}^{2}+\left\|P_{K, \mathrm{RM}} \lambda-\Pi_{h} \lambda\right\|_{0, \partial K}^{2} \\
& \lesssim h_{K}^{4}\left|\Pi_{h} \lambda\right|_{2, \partial K}^{2}+\sum_{K^{\prime} \in \omega_{K}}\left\|\lambda-P_{K^{\prime}, \mathrm{RM}} \lambda\right\|_{0, \partial K^{\prime}}^{2} \quad(\text { by } 4.28) \\
& \lesssim h_{K}\left\|\epsilon\left(\Pi_{h} \lambda\right)\right\|_{0, K}^{2}+\sum_{K^{\prime} \in \omega_{K}}\left\|\lambda-P_{K^{\prime}, \mathrm{RM}} \lambda\right\|_{0, \partial K^{\prime}}^{2} .
\end{aligned}
$$

This completes the proof.

Let $\left\{W_{i}\right\}_{i=1}^{J}$ be the local spaces of $W_{h}$ associated with the overlapping domain decomposition $\left\{\Omega_{i}\right\}_{i=1}^{J}$. According to [49], we have the following lemma.

Lemma 4.8. For any $w_{h} \in W_{h}$, there exists a decomposition $w_{h}=\tilde{I}_{H}^{h} w_{H}+$ $\sum_{i=1}^{J} w_{i}$, such that $w_{H} \in W_{H}, w_{i} \in W_{i}$, and

$$
\left\|w_{H}\right\|_{A_{H}}^{2}+\sum_{i=1}^{J}\left\|w_{i}\right\|_{A_{h}}^{2} \lesssim \frac{H^{2}}{\delta^{2}}\left\|w_{h}\right\|_{A_{h}}^{2} .
$$

Theorem 4.9. For any $\lambda \in M_{h, k+1}$, there exists a decomposition $\lambda=I_{H}^{h} w_{H}+$ $\sum_{i=1}^{J} \lambda_{i}$ such that $w_{H} \in W_{H}, \lambda_{i} \in M_{i}$, and

$$
\left\|w_{H}\right\|_{A_{H}}^{2}+\sum_{i=1}^{J}\left\|\lambda_{i}\right\|_{S}^{2} \lesssim \frac{H^{2}}{\delta^{2}}\|\lambda\|_{S}^{2} .
$$

Proof. We first split $\lambda$ into two components

$$
\lambda=Q_{h} \underbrace{\Pi_{h} \lambda}_{w_{h}}+\underbrace{\left(\lambda-Q_{h} \Pi_{h} \lambda\right)}_{\lambda_{0}} .
$$

According to the Lemma 4.7, we know that

$$
\lambda_{0} \in M_{h, 0}^{\perp} \quad \text { and } \quad\left\|\lambda_{0}\right\|_{0}^{2} \lesssim h\|\lambda\|_{S}^{2} .
$$

Here, $M_{h, 0}^{\perp}$ is the $L^{2}$ orthogonal complement of $M_{h, 0}$ in the space $M_{h, k+1}$. Denote the $L^{2}$ projection on $M_{h, 0}^{\perp}$ by $Q_{0}^{\perp}$. Let $w_{h}=\tilde{I}_{H}^{h} w_{H}+\sum_{i=1}^{J} w_{i}$ be the decomposition in Lemma 4.8. We define the $\lambda_{i}$ as

$$
\lambda_{i}=Q_{h} w_{i}+Q_{0}^{\perp}\left(\theta_{i} \lambda_{0}\right) \quad j=1,2, \cdots, J
$$


Thus, $\lambda=I_{H}^{h} w_{H}+\sum_{i=1}^{J} \lambda_{i}$. By the property of the partition of unity, Theorem 3.7. Lemma 4.4, 4.7, and 4.8, we have

$$
\begin{aligned}
\sum_{i=1}^{J}\left\|\lambda_{i}\right\|_{S}^{2} & =\sum_{i=1}^{J} \sum_{K \in \mathcal{T}_{h} \cap \Omega_{i}}\left\|\lambda_{i}\right\|_{S, K}^{2} \\
& \lesssim \sum_{i=1}^{J}\left\|Q_{h} w_{i}\right\|_{S}^{2}+\sum_{i=1}^{J} \sum_{K \in \mathcal{T}_{h} \cap \Omega_{i}}\left\|Q_{0}^{\perp}\left(\theta_{i} \lambda_{0}\right)\right\|_{S, K}^{2} \\
& \lesssim \sum_{i=1}^{J}\left\|Q_{h} w_{i}\right\|_{S}^{2}+\sum_{i=1}^{J} \sum_{K \in \mathcal{T}_{h} \cap \Omega_{i}} h_{K}^{-1}\left\|Q_{0}^{\perp}\left(\theta_{i} \lambda_{0}\right)\right\|_{0, \partial K}^{2} \quad \text { (by 3.16) } \\
& \lesssim \sum_{i=1}^{J}\left\|Q_{h} w_{i}\right\|_{S}^{2}+h^{-1}\left\|\lambda_{0}\right\|_{0}^{2} \quad \text { (by Lemma 4.4 and 4.31) } \\
& \lesssim \frac{H^{2}}{\delta^{2}}\left\|w_{h}\right\|_{A_{h}}^{2}+h^{-1}\left\|\lambda_{0}\right\|_{0}^{2} \quad \text { (by 4.24 and 4.25) } \\
& \lesssim \frac{H^{2}}{\delta^{2}}\|\lambda\|_{S}^{2},
\end{aligned}
$$

and

$$
\left\|w_{H}\right\|_{A_{H}} \lesssim \frac{H^{2}}{\delta^{2}}\left\|w_{h}\right\|_{A_{h}} \lesssim \frac{H^{2}}{\delta^{2}}\|\lambda\|_{S}
$$

This completes the proof.

\section{Numerical Examples}

In this section, we give several numerical examples to present the optimal convergence order of the hybridized mixed discretization as well as the uniform convergence of the iterative solvers. All the numerical experiments are implemented using the iFEM package [19.

5.1. Convergence Order Tests. To verify the convergence order for the discretization, we take the domain to be unit square $\Omega=(0,1)^{2}$ and choose the data with the exact solution given by

$$
u=\left(\begin{array}{c}
\mathrm{e}^{x-y} x y(1-x)(1-y) \\
\sin (\pi x) \sin (\pi y)
\end{array}\right)
$$

We apply a homogeneous boundary condition that $u=0$ on $\partial \Omega$. The Lamé constants are set as $\tilde{\mu}=1 / 2$ and $\tilde{\lambda}=1$. The exact stress function $\sigma$ and the load function $f$ can be analytically derived from the 2.1 for a given $u$. We use the MATLAB backslash solver for the system of the multiplier if the grid is singular-vertex free, and the conjugate gradient method with diagonal preconditioning otherwise.

Example 5.1 (Lowest order method on macro-simplex grid). Our first numerical example is carried out on the macro-simplex grid, which can be obtained from any triangulation by connecting the vertices of each triangle to the barycenter, thereby subdividing the triangle into three, see Figure 3.

After computing (3.3) for various values of $h$, we calculate the errors between the exact solution and the discrete solution and report them in Table 2. The table indicates the optimal convergence orders of $\mathcal{O}(h)$ for both stress and displacement in the $H(\operatorname{div})$ and $L^{2}$ norm, respectively. 

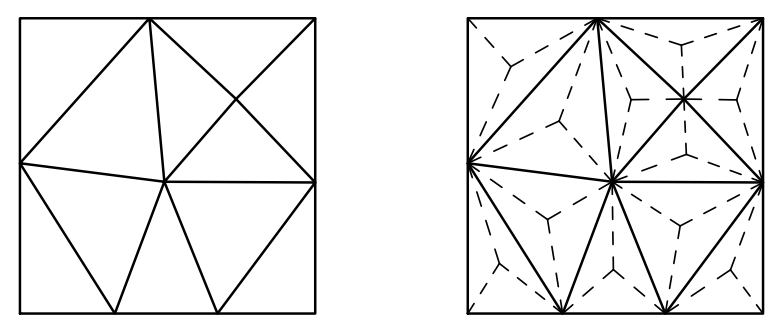

FiguRE 3. Unstructured grid and a typical macro-simplex grid.

TABLE 2. Errors and observed convergence orders on macrosimplex grids, $k=0$.

\begin{tabular}{c|cc|cc|cc}
\hline $1 / h$ & $\left\|u-u_{h}\right\|_{0}$ & Order & $\left\|\boldsymbol{\sigma}-\boldsymbol{\sigma}_{h}\right\|_{0}$ & Order & $\left\|\operatorname{div} \boldsymbol{\sigma}-\operatorname{div} \boldsymbol{\sigma}_{h}\right\|_{0}$ & Order \\
\hline 4 & $9.5309 \mathrm{e}-2$ & - & $2.0147 \mathrm{e}-1$ & - & $2.6589 \mathrm{e}-0$ & - \\
8 & $4.5289 \mathrm{e}-2$ & 1.07 & $4.9971 \mathrm{e}-2$ & 2.01 & $1.2995 \mathrm{e}-0$ & 1.03 \\
16 & $2.2009 \mathrm{e}-2$ & 1.04 & $1.2357 \mathrm{e}-2$ & 2.01 & $6.3735 \mathrm{e}-1$ & 1.02 \\
32 & $1.0976 \mathrm{e}-2$ & 1.00 & $3.1761 \mathrm{e}-3$ & 1.96 & $3.1827 \mathrm{e}-1$ & 1.00 \\
64 & $5.4797 \mathrm{e}-3$ & 1.00 & $8.0961 \mathrm{e}-4$ & 1.97 & $1.5892 \mathrm{e}-1$ & 1.00 \\
\hline
\end{tabular}

Example 5.2 (High order method). We apply the finite element method with $k=2$ for the high order case, which is the lowest order method that works for any $2 \mathrm{D}$ regular grid. The computations are performed on both the uniform grid and crisscross grid as depicted in Figure 4.

We list the errors and observed convergence orders of the computed solution on the uniform grid in Table 3. It clearly indicates that $\left\|u-u_{h}\right\|_{0}=\mathcal{O}\left(h^{3}\right)$ and $\left\|\boldsymbol{\sigma}-\boldsymbol{\sigma}_{h}\right\|_{H(\text { div })}=\mathcal{O}\left(h^{3}\right)$ which agrees with Theorem 2.5. In addition, we observe that $\left\|\boldsymbol{\sigma}-\boldsymbol{\sigma}_{h}\right\|_{0}=\mathcal{O}\left(h^{4}\right)$. Similar results can be observed on the crisscross grid as shown in Table 4 . As discussed in Section 3 , the singular vertices do not affect the well-posedness of the original saddle point problem but only results in a SPSD system for the Lagrange multiplier, which can be solved efficiently by the Krylov solvers.
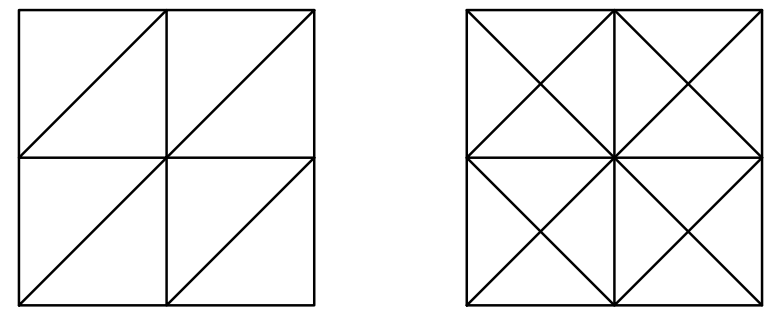

FiguRE 4. Uniform grid and crisscross grid

5.2. Iterative Solver Tests. In this subsection, we investigate the robustness of our iterative solvers with respect to both the mesh size $h$ and Poisson's ratio $\tilde{\nu}$. In all the numerical experiments below, we choose the data such that the exact solution is given by (5.1). High-order discretization of $k=2$ is applied on the 
TABLE 3. Errors and observed convergence orders on uniform grids, $k=2$.

\begin{tabular}{c|cc|cc|cc}
\hline $1 / h$ & $\left\|u-u_{h}\right\|_{0}$ & Order & $\left\|\boldsymbol{\sigma}-\boldsymbol{\sigma}_{h}\right\|_{0}$ & Order & $\left\|\operatorname{div} \boldsymbol{\sigma}-\operatorname{div} \boldsymbol{\sigma}_{h}\right\|_{0}$ & Order \\
\hline 4 & $2.1758 \mathrm{e}-3$ & - & $2.0260 \mathrm{e}-3$ & - & $6.2558 \mathrm{e}-2$ & - \\
8 & $2.7561 \mathrm{e}-4$ & 2.98 & $1.5145 \mathrm{e}-4$ & 3.89 & $7.9274 \mathrm{e}-3$ & 2.98 \\
16 & $3.4569 \mathrm{e}-5$ & 2.99 & $9.7454 \mathrm{e}-6$ & 3.95 & $9.9431 \mathrm{e}-4$ & 2.99 \\
32 & $4.3248 \mathrm{e}-6$ & 2.99 & $6.1737 \mathrm{e}-7$ & 3.98 & $1.2439 \mathrm{e}-4$ & 3.00 \\
64 & $5.4072 \mathrm{e}-7$ & 3.00 & $3.8838 \mathrm{e}-8$ & 3.99 & $1.5552 \mathrm{e}-5$ & 3.00 \\
\hline
\end{tabular}

TABLE 4. Errors and observed convergence orders on crisscross grids, $k=2$.

\begin{tabular}{c|cc|cc|cc}
\hline $1 / h$ & $\left\|u-u_{h}\right\|_{0}$ & Order & $\left\|\boldsymbol{\sigma}-\boldsymbol{\sigma}_{h}\right\|_{0}$ & Order & $\|$ div $\boldsymbol{\sigma}-$ div $\boldsymbol{\sigma}_{h} \|_{0}$ & Order \\
\hline 4 & $5.7633 \mathrm{e}-4$ & - & $3.1371 \mathrm{e}-4$ & - & $1.7027 \mathrm{e}-2$ & - \\
8 & $7.2355 \mathrm{e}-5$ & 2.99 & $2.0057 \mathrm{e}-5$ & 3.96 & $2.1361 \mathrm{e}-3$ & 2.99 \\
16 & $9.0541 \mathrm{e}-6$ & 2.99 & $1.2672 \mathrm{e}-6$ & 3.98 & $2.6726 \mathrm{e}-4$ & 2.99 \\
32 & $1.1320 \mathrm{e}-6$ & 3.00 & $7.9629 \mathrm{e}-8$ & 3.99 & $3.3416 \mathrm{e}-4$ & 3.00 \\
64 & $1.4151 \mathrm{e}-7$ & 3.00 & $4.9899 \mathrm{e}-9$ & 4.00 & $4.1772 \mathrm{e}-5$ & 3.00 \\
\hline
\end{tabular}

uniform grids. The Lamé constants are set as $\tilde{\mu}=1 / 2$ and

$$
\tilde{\lambda}=\frac{\tilde{\nu}}{1-2 \tilde{\nu}}
$$

where $\tilde{\nu}$ represents the Poisson's ratio that goes to 0.5 when the material becomes increasingly incompressible.

We run the various preconditioning Conjugate Gradient (PCG) computations with zero initial guess and a stopping criterion whereby the relative residual is smaller than $10^{-6}$. We verify the reasonableness of our choices for Schwarz smoother, intergrid transfer operator and coarse solvers in the following numerical experiments.

Example 5.3 (One-level Schwarz preconditioner). This example is to verify the $\tilde{\nu}$-independent property of the Schwarz method on the fine grid. Clearly any local space defined on the vertex patch (edges that share the same vertex) belongs to one subspace defined in 4.2 at least. Hence, the corresponding Schwarz method would be uniform with respective to $\tilde{\nu}$. We also test the other two choices of the space decompositions with supported sets on edge patches and element patches, respectively.

Table 5 presents the number of iterations of PCG with symmetrized multiplicative Schwarz preconditioner for different decompositions. The mesh size is set as $h=1 / 4$. Only the decomposition consisting of vertex patches provides a $\tilde{\nu}$ independent method.

Example 5.4 (Two-level Schwarz preconditioners). We now validate the robustness of the two-level Schwarz preconditioner. We note that the $\mathcal{P}_{2}$ Lagrange finite element space $W_{H}$ is used as coarse space due to its d.o.f. that preserve rigidbody motion as well as the moments on the edges, see Lemma 4.7. The fine grid $\mathcal{T}_{h}=\left\{K_{h}\right\}$ is refined uniformly from the coarse grid $\mathcal{T}_{H}=\left\{K_{H}\right\}$. Hence, the overlap is set as $\delta=h$ and the ratio $H / \delta=2$. The intergrid transfer operator is defined as $I_{H}^{h}=Q_{h} \tilde{I}_{H}^{h}$ in 4.8 . 
TABLE 5. Number of iterations of PCG: One-level multiplicative Schwarz preconditioner with subspaces supported on edges, elements, and vertex patches.

\begin{tabular}{|c|c|c|c|c|c|c|}
\hline Subdomains $\tilde{\nu}$ & 0.49 & 0.499 & 0.4999 & 0.49999 & 0.499999 & 0.4999999 \\
\hline Edges & 36 & 59 & 79 & 109 & 131 & 154 \\
\hline Elements & 15 & 24 & 33 & 45 & 54 & 62 \\
\hline Vertex Patches & 10 & 12 & 13 & 13 & 14 & 14 \\
\hline
\end{tabular}

Table 6 lists the number of iterations of PCG using the additive Schwarz preconditioner 4.4 and the corresponding symmetrized multiplicative Schwarz preconditioner. This result clearly shows the robustness of the Schwarz preconditioner in agreement with the Theorem 4.1 .

TABLE 6. Number of iterations of PCG: Two-level additive Schwarz preconditioner (left) and symmetrized multiplicative Schwarz preconditioner (right)

\begin{tabular}{|c|c|c|c|c|c|c|}
\hline $1 / h{ }^{\nu}$ & 0.49 & 0.499 & 0.4999 & 0.49999 & 0.499999 & 0.4999999 \\
\hline 4 & 17,3 & 18,4 & 21,4 & 23,4 & 23,4 & 23,4 \\
\hline 8 & 17,4 & 20,4 & 25,4 & 27,5 & 28,5 & 29,5 \\
\hline 16 & 18,4 & 20,4 & 26,5 & 28,5 & 29,5 & 29,5 \\
\hline 32 & 18,4 & 20,4 & 25,5 & 27,5 & 28,5 & 29,5 \\
\hline
\end{tabular}

Example 5.5 (Multilevel preconditioner). We test the scalability of a multilevel preconditioner. In this test, we use $W_{H}$ (i.e., continuous space of piecewise $\left.\left(\mathcal{P}_{2}\right)^{2}\right)$ as the coarse space. The intergrid transfer operator and the overlapping subdomains and are the same as those of the second test. Instead of using an exact solver for $A_{H}$, we solve the coarse problem approximately using a W-2-2 cycle in [48. Table 7 shows the uniform convergence of the multilevel symmetrized multiplicative preconditioner.

\section{Concluding Remarks}

Motivated by the critical observation on the inter-element jump of the piecewise discontinuous symmetric-matrix-valued polynomials, we propose a family of hybridizable mixed finite elements for linear elasticity. These methods extend the works in [11, 5, 36, 32] by relaxing the continuity of the discrete stress on the grid

TABLE 7. Number of iterations of PCG, multilevel symmetrized multiplicative preconditioner.

\begin{tabular}{|c|c|c|c|c|c|c|}
\hline $1 / h^{\tilde{\nu}}$ & 0.49 & 0.499 & 0.4999 & 0.49999 & 0.499999 & 0.4999999 \\
\hline 4 & 4 & 5 & 5 & 5 & 5 & 5 \\
\hline 8 & 4 & 6 & 7 & 7 & 7 & 7 \\
\hline 16 & 5 & 6 & 7 & 7 & 7 & 7 \\
\hline 32 & 5 & 6 & 7 & 7 & 7 & 7 \\
\hline
\end{tabular}


vertices while preserving the symmetry and $H$ (div) conformity in stress approximation. By hybridization, the solution cost for our discretization is dominated by the cost of solving the global system of the Lagrange multiplier. To develop robust solvers, we adopt the Schwarz method on the fine grid and the primal method as a coarse problem. The key to proving the uniform convergence of our iterative solvers is the construction of the interpolation operator $I_{h}$, which is stable with the approximation property (see Lemma 4.7). The new discretization, which preserves the physical structure of stress, along with the robust solver provides a new competitive approach for stress analysis in computational structure mechanics.

\section{Appendix. Proofs of Lemmas 3.4 and 3.9}

Proof of Lemma 3.4. Denote the set of all $k+1$ degree Lagrange nodes in $\mathcal{T}_{h}$ by $A_{h, k+1}$. For any $K \in \mathcal{T}_{h}$ and $a \in A_{h, k+1} \cap \bar{K}$, let $\varphi_{a}^{K}$ be the Lagrange nodal basis in $K$, with zero extension in $\mathcal{T}_{h} \backslash K$. Further, for any $F \in \mathcal{F}_{h}^{i}$ and $a \in A_{h, k+1} \cap \bar{F}$, let $\psi_{a}^{F}$ be the dual basis of the degree $k+1$ Lagrange basis that

$$
\left\langle\psi_{a^{\prime}}^{F}, \varphi_{a}^{K}\right\rangle_{F}=\delta_{a, a^{\prime}} \quad \text { and }\left.\quad \psi_{a^{\prime}}^{F}\right|_{\mathcal{F}_{h} \backslash F}=0 .
$$

For any $a \in A_{h, k+1}$, define the local spaces

$$
\begin{aligned}
\Sigma_{h, k+1, a}^{-1} & :=\operatorname{span}\left\{\varphi_{a}^{K} T_{i j} \mid 1 \leq i \leq j \leq n, \bar{K} \ni a\right\}, \\
M_{h, k+1, a} & :=\operatorname{span}\left\{\psi_{a}^{F} e_{i} \mid 1 \leq i \leq n, F \in \mathcal{F}_{h}^{i}, \bar{F} \ni a\right\},
\end{aligned}
$$

where $\left\{e_{i} \mid 1 \leq i \leq n\right\}$ is the basis of $\mathbb{R}^{n}$ and $\left\{T_{i j}=\frac{1}{2}\left(e_{i} e_{j}^{T}+e_{j} e_{i}^{T}\right) \mid 1 \leq i \leq j \leq n\right\}$ is the basis of $\mathbb{S}$. Clearly,

$$
\Sigma_{h, k+1}^{-1}=\bigoplus_{a \in A_{h, k+1}} \Sigma_{h, k+1, a}^{-1} \quad \text { and } \quad M_{h, k+1}=\bigoplus_{a \in A_{h, k+1}} M_{h, k+1, a} .
$$

Moreover, if $a \neq a^{\prime}$ and $\mu \in \mathcal{C}\left(\Sigma_{h, k+1, a}^{-1}\right) \cap \mathcal{C}\left(\Sigma_{h, k+1, a^{\prime}}^{-1}\right)$, then $\mu$ vanishes at all the Lagrange nodes on the edges. This implies that $\mu=0$, namely

$$
\mathcal{C}\left(\Sigma_{h, k+1, a}^{-1}\right) \cap \mathcal{C}\left(\Sigma_{h, k+1, a^{\prime}}^{-1}\right)=\{0\} \quad \text { if } a \neq a^{\prime} .
$$

Hence, we have

$$
\mathrm{R}(\mathcal{C})=\mathcal{C}\left(\Sigma_{h, k+1}^{-1}\right)=\bigoplus_{a \in A_{h, k+1}} \mathcal{C}\left(\Sigma_{h, k+1, a}^{-1}\right) .
$$

Therefore, $\mathrm{R}(\mathcal{C})$ has local basis since $\mathcal{C}\left(\Sigma_{h, k+1, a}^{-1}\right)$ is locally supported for any $a \in$ $A_{h, k+1}$.

Next, we construct the local basis for $\mathrm{R}(\mathcal{C})^{\perp}$. Let

$$
M_{h, k+1, a, \perp}:=\left\{\mu_{a} \in M_{h, k+1, a} \mid\left\langle\mu_{a},\left[\boldsymbol{\tau}_{a}\right]\right\rangle_{\mathcal{F}_{h}^{i}}=0 \quad \forall \boldsymbol{\tau}_{a} \in \Sigma_{h, k+1, a}^{-1}\right\} .
$$

If $a \neq a^{\prime}$, we further have

$$
\langle\mu, \mathcal{C} \boldsymbol{\tau}\rangle_{\mathcal{F}_{h}^{i}}=0 \quad \forall \mu \in M_{h, k+1, a}, \boldsymbol{\tau} \in \Sigma_{h, k+1, a^{\prime}}^{-1}
$$

Hence, we have $M_{h, k+1, a, \perp} \subset \mathrm{R}(\mathcal{C})^{\perp}$ and

$$
\mathrm{R}(\mathcal{C})^{\perp}=\bigoplus_{a \in A_{h, k}} M_{h, k+1, a, \perp}
$$

Therefore, the local basis $\left\{\psi_{1}, \psi_{2}, \cdots, \psi_{N_{2}}\right\}$ of $\mathrm{R}(\mathcal{C})^{\perp}$ comes from the union of the basis of $M_{h, k+1, a, \perp}$ for all $a \in A_{h, k}$. 
In addition, the basis of $M_{h, k+1, a, \perp}$ can be computed locally according to its definition 6.3. In particular, $M_{h, k+1, a, \perp}$ is nontrivial for the $2 \mathrm{D}$ case only if $a$ is an interior singular vertex. Thus, if there is no interior singular vertex in $\mathcal{T}_{h}$, then $\mathrm{R}(\mathcal{C})^{\perp}=\{0\}$, or $M_{h, k+1}=\mathrm{R}(\mathcal{C})=\bigoplus_{a \in A_{h, k+1}} \mathcal{C}\left(\Sigma_{h, k+1, a}^{-1}\right)$. Further, a direct calculation shows that

$$
\mathcal{C}\left(\Sigma_{h, k+1, a}^{-1}\right)= \begin{cases}\operatorname{span}\left\{\varphi_{a}^{F} e_{i} \mid 1 \leq i \leq 2, F \in \mathcal{F}_{h}^{i}, \bar{F} \ni a\right\} & a \in \overline{\mathcal{F}}_{h}^{i} \\ \{0\} & a \notin \overline{\mathcal{F}}_{h}^{i}\end{cases}
$$

where $\varphi_{a}^{F}$ denotes the Lagrange nodal basis on $F$. Therefore, we can choose a special basis of $\mathrm{R}(\mathcal{C})$ as

$$
M_{h, k+1}=\bigoplus_{a \in \bar{F}_{h}^{i} \cap A_{h, k+1}} \operatorname{span}\left\{\varphi_{a}^{F} e_{i} \mid 1 \leq i \leq 2, F \in \mathcal{F}_{h}^{i}, \bar{F} \ni a\right\} .
$$

The mass matrix under the special basis 6.4 is the diagonal block matrix whose diagonal block entry is the local mass matrix under the Lagrange nodal basis on $F$. Hence, the mass matrix $M$ is well-conditioned because the local mass matrix is well conditioned for the Lagrange nodal basis, which gives rise to (3.7). This completes the proof.

Proof of Lemma 3.9. In light of $\sqrt{6.2}$ in the proof of Lemma 3.4, there exists a Lagrange node $a \in A_{h, k+1}$ such that $\varphi_{i} \in \mathcal{C}\left(\Sigma_{h, k+1, a}^{-1}\right)$. Further, we have

$$
\varphi_{i}=\left.\omega \varphi_{a}\right|_{\mathcal{F}_{h}},
$$

where $\varphi_{a}$ is the Lagrange nodal basis function at the node $a$ and $\omega \in L^{2}\left(\mathcal{F} ; \mathbb{R}^{2}\right)$ is piecewise constant and $\operatorname{supp}(\omega) \subset\left\{F \in \mathcal{F}_{h}^{i} \mid \bar{F} \ni a\right\}$. Next, we construct $\boldsymbol{\tau}_{i} \in \Sigma_{h, k+1}^{-1}$ case by case according to the location of $a$. Clearly, if $a$ is not located on the $\overline{\mathcal{F}}_{h}^{i}$, then $\mathcal{C}\left(\Sigma_{h, k+1, a}^{-1}\right)=\{0\}$. Hence, we only need to consider the following two cases: Internal Lagrange node on $F \in \mathcal{F}_{h}^{i}$, or vertex of $\mathcal{T}_{h}$. We first state a useful tool for the analysis: For any given vectors $v, w \in \mathbb{R}^{2}$, there exists $T \in \mathbb{S}$ such that

$$
T v=w \quad \text { and } \quad\|T\|_{l^{2}} \leq \sqrt{2} \frac{\|w\|_{l^{2}}}{\|v\|_{l^{2}}}
$$

A straightforward calculation shows that $T$ in 6.5 can be chosen as

$$
T=\frac{w_{1}}{\|v\|_{l^{2}}^{2}}\left(\begin{array}{cc}
v_{1} & v_{2} \\
v_{2} & -v_{1}
\end{array}\right)+\frac{w_{2}}{\|v\|_{l^{2}}^{2}}\left(\begin{array}{cc}
-v_{2} & v_{1} \\
v_{1} & v_{2}
\end{array}\right)
$$

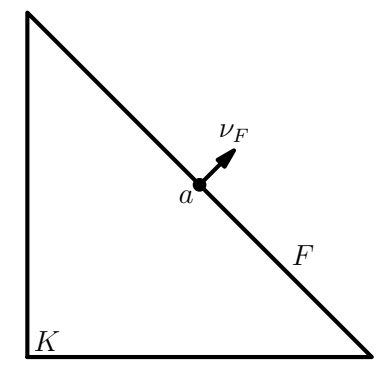

Figure 5. Internal Lagrange node on edge $F$. 
Case 1: Internal Lagrange node of $F \in \mathcal{F}_{h}^{i}$. First, we select an element $K$ such that $F \in \bar{K}$ (cf. Figure 5). By virtue of (6.5), there exists $T \in \mathbb{S}$ such that

$$
T \nu_{F}=\left.\omega\right|_{F} \text { and }\|T\|_{l^{2}} \lesssim\left\|\left.\omega\right|_{F}\right\|_{l^{2}}
$$

From the definition of $\Sigma_{h, k+1, a}^{-1}$ in (6.1), let $\boldsymbol{\tau}_{i}=\varphi_{a}^{K} T \in \Sigma_{h, k+1, a}^{-1}$. Then,

$\left[\boldsymbol{\tau}_{i}\right]_{F}=\left.\varphi_{i}\right|_{F} \quad \forall F \in \mathcal{F}_{h} \quad$ and $\quad\left\|\boldsymbol{\tau}_{i}\right\|_{0}^{2}=\left\|\varphi_{a}\right\|_{0, K}^{2}\|T\|_{l^{2}}^{2} \lesssim h\left\|\varphi_{a}\right\|_{0, F}^{2}\left\|\left.\omega\right|_{F}\right\|_{l^{2}}^{2}=h\left\|\varphi_{i}\right\|_{0}^{2}$.

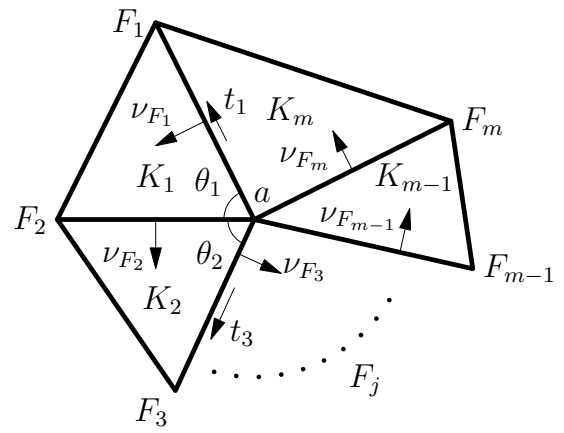

(A) Internal vertex

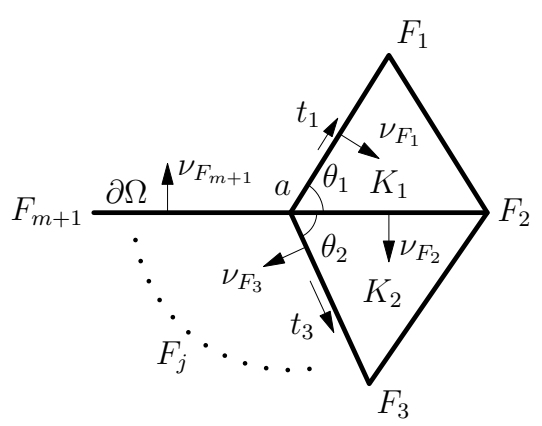

(в) Boundary vertex

Figure 6. Vertex of $\mathcal{T}_{h}$.

Case 2: Vertex of $\mathcal{T}_{h}$. Suppose that there are $m(\geq 2)$ elements meeting at the vertex $a$. Since $\kappa \geq \kappa_{0}>0$, there exist two adjacent elements (without loss of generality, denoted by $K_{1}$ and $K_{2}$ ), such that the angles $\theta_{1}$ and $\theta_{2}$ satisfying $\left|\theta_{1}+\theta_{2}-\pi\right| \geq \kappa_{0}$, (cf. Figure 6). The edges that contain $a$ are denoted by $F_{j}, 1 \leq j \leq m$ if $a$ is an internal vertex, and $1 \leq j \leq m+1$ otherwise. If $a$ is a boundary vertex, we further set $F_{1}, F_{m+1} \in \mathcal{F}_{h}^{\partial}$, which is feasible because $\kappa(a) \geq \kappa_{0}>0$.

If $a$ is an internal vertex, let $F_{m+1}=F_{1}$ and $\nu_{F_{m+1}}=\nu_{F_{1}}$. By virtue of (6.5), there exists $T_{m} \in \mathbb{S}$ such that

$$
T_{m} \nu_{F_{m+1}}=\left.\omega\right|_{F_{m+1}} \quad \text { and } \quad\left\|T_{m}\right\|_{l^{2}} \lesssim\left\|\left.\omega\right|_{F_{m+1}}\right\|_{l^{2}} .
$$

Note that $T_{m}=\mathbf{0} \in \mathbb{S}$ if $a$ is a boundary vertex. Recursively for $j=m-1, m-$ $2, \cdots, 2$, there exist $T_{j} \in \mathbb{S}$ on $K_{j}$ such that

$$
T_{j} \nu_{F_{j+1}}=\left.\omega\right|_{F_{j+1}}+T_{j+1} \nu_{F_{j+1}} \quad \text { and } \quad\left\|T_{j}\right\|_{l^{2}} \lesssim\left\|\left.\omega\right|_{F_{j+1}}\right\|_{l^{2}}+\left\|T_{j+1}\right\|_{l^{2}} \lesssim \sum_{s=j}^{m+1}\left\|\left.\omega\right|_{F_{s}}\right\|_{l^{2}} \text {. }
$$

Since $\left.\omega\right|_{F_{0}}=0$ if $a$ is a boundary vertex, we simply set $T_{1}=\mathbf{0} \in \mathbb{S}$.

Next, we find two symmetric matrices $\tilde{T}_{1}=c_{1} t_{1} t_{1}^{T}$ and $\tilde{T}_{2}=c_{2} t_{3} t_{3}^{T}$ on $K_{1}$ and $K_{2}$, respectively. Here, $t_{1}, t_{3}$ are the unit tangential vectors of $F_{1}$ and $F_{3}$, respectively (cf. Figure 6). The coefficients $c_{1}, c_{2}$ are determined by

$$
\tilde{T}_{1} \nu_{F_{2}}-\tilde{T}_{2} \nu_{F_{2}}=\left.\omega\right|_{F_{2}}+T_{2} \nu_{F_{2}},
$$

i.e.

$$
-\left(t_{1}, t_{3}\right)\left(\begin{array}{c}
c_{1} \sin \theta_{1} \\
c_{2} \sin \theta_{2}
\end{array}\right)=\left.\omega\right|_{F_{2}}+T_{2} \nu_{F_{2}} .
$$


Since $\left|\theta_{1}+\theta_{2}-\pi\right| \geq \kappa_{0}$, we have $\left|\operatorname{det}\left(t_{1}, t_{3}\right)\right|=\left|t_{1} \times t_{3}\right|=\left|\sin \left(\theta_{1}+\theta_{2}\right)\right| \geq \sin \left(\kappa_{0}\right)$. Thus, the matrix $\left(t_{1}, t_{3}\right)$ is invertible. Moreover, we have $\left|\left(t_{1}, t_{3}\right)^{-1}\right|_{\infty} \lesssim \sin ^{-1}\left(\kappa_{0}\right)$ and, by the shape regularity of grids, $\left|\sin \theta_{1}\right|$ and $\left|\sin \theta_{2}\right|$ are bounded uniformly away from zero. Thus,

$$
\left\|\tilde{T}_{1}\right\|_{l^{2}}^{2}+\left\|\tilde{T}_{1}\right\|_{l^{2}}^{2} \lesssim c_{1}^{2}+c_{2}^{2} \lesssim \sin ^{-2}\left(\kappa_{0}\right)\left\|\left.\omega\right|_{F_{2}}+T_{2} \nu_{F_{2}}\right\|_{l^{2}}^{2} \lesssim \sin ^{-2}\left(\kappa_{0}\right) \sum_{j=1}^{m+1}\left\|\left.\omega\right|_{F_{j}}\right\|_{l^{2}}^{2} .
$$

In light of $6.6,6.7)$, and 6.8 , let

$$
\left.\boldsymbol{\tau}_{i}\right|_{K_{j}}= \begin{cases}\varphi_{a}^{K_{j}}\left(T_{j}+\tilde{T}_{j}\right) & j=1,2 \\ \varphi_{a}^{K_{j}} T_{j} & 3 \leq j \leq m\end{cases}
$$

Then, we have

$$
\left.\left[\boldsymbol{\tau}_{i}\right]\right|_{F}=\left.\varphi_{i}\right|_{F} \quad \forall F \in \mathcal{F}_{h}, \quad \text { and } \quad\left\|\boldsymbol{\tau}_{i}\right\|_{0}^{2} \lesssim h \sin ^{-2}\left(\kappa_{0}\right)\left\|\varphi_{i}\right\|_{0}^{2} .
$$

This completes the proof.

\section{REFERENCES}

1. Scot Adams and Bernardo Cockburn, A mixed finite element method for elasticity in three dimensions, Journal of Scientific Computing 25 (2005), no. 3, 515-521.

2. Mohamed Amara and Jean-Marie Thomas, Equilibrium finite elements for the linear elastic problem, Numerische Mathematik 33 (1979), no. 4, 367-383.

3. Paola F Antonietti, Marco Verani, and Ludmil Zikatanov, A two-level method for mimetic finite difference discretizations of elliptic problems, Computers \& Mathematics with Applications 70 (2015), no. 11, 2674-2687.

4. Douglas N. Arnold and Gerard Awanou, Rectangular mixed finite elements for elasticity, Mathematical Models and Methods in Applied Sciences 15 (2005), no. 09, 1417-1429.

5. Douglas N. Arnold, Gerard Awanou, and Ragnar Winther, Finite elements for symmetric tensors in three dimensions, Mathematics of Computation 77 (2008), no. 263, 1229-1251.

6. Douglas N. Arnold and Franco Brezzi, Mixed and nonconforming finite element methods: implementation, postprocessing and error estimates, RAIRO-Modélisation mathématique et analyse numérique 19 (1985), no. 1, 7-32.

7. Douglas N. Arnold, Jim Douglas Jr, and Chaitan P Gupta, A family of higher order mixed finite element methods for plane elasticity, Numerische Mathematik 45 (1984), no. 1, 1-22.

8. Douglas N. Arnold, Richard Falk, and Ragnar Winther, Mixed finite element methods for linear elasticity with weakly imposed symmetry, Mathematics of Computation 76 (2007), no. 260, 1699-1723.

9. Douglas N. Arnold, Richard S Falk, and Ragnar Winther, Finite element exterior calculus, homological techniques, and applications, Acta numerica 15 (2006), no. 1, 1-155.

10. Douglas N. Arnold and Jinshui Qin, Quadratic velocity/linear pressure stokes elements, Advances in computer methods for partial differential equations 7 (1992), 28-34.

11. Douglas N. Arnold and Ragnar Winther, Mixed finite elements for elasticity, Numerische Mathematik 92 (2002), no. 3, 401-419.

12. _ Nonconforming mixed elements for elasticity, Mathematical Models and Methods in Applied Sciences 13 (2003), no. 03, 295-307.

13. Gerard Awanou, A rotated nonconforming rectangular mixed element for elasticity, Calcolo 46 (2009), no. 1, 49-60.

14. Daniele Boffi, Franco Brezzi, and Michel Fortin, Reduced symmetry elements in linear elasticity, Commun. Pure Appl. Anal 8 (2009), no. 1, 95-121.

15. Susanne Brenner and Ridgway Scott, The mathematical theory of finite element methods, vol. 15, Springer Science \& Business Media, 2007.

16. Susanne C Brenner, Multigrid methods for parameter dependent problems, RAIROModélisation mathématique et analyse numérique 30 (1996), no. 3, 265-297.

17. Franco Brezzi, Jim Douglas Jr, and L Donatella Marini, Two families of mixed finite elements for second order elliptic problems, Numerische Mathematik 47 (1985), no. 2, 217-235. 
18. Franco Brezzi and Michel Fortin, Mixed and hybrid finite element methods, vol. 15, Springer Science \& Business Media, 2012.

19. Long Chen, iFEM: an innovative finite element methods package in MATLAB, Preprint, University of Maryland (2008).

20. Long Chen, Jun $\mathrm{Hu}$, and Xuehai Huang, Fast auxiliary space preconditioner for linear elasticity in mixed form, arXiv preprint arXiv:1604.02568 (2016).

21. Durkbin Cho, Jinchao Xu, and Ludmil Zikatanov, New estimates for the rate of convergence of the method of subspace corrections, Numer. Math. Theory Methods Appl 1 (2008), no. 1, $44-56$.

22. Bernardo Cockburn, O Dubois, Jay Gopalakrishnan, and S Tan, Multigrid for an HDG method, IMA Journal of Numerical Analysis 34 (2014), no. 4, 1386-1425.

23. Bernardo Cockburn, Jayadeep Gopalakrishnan, and Johnny Guzmán, A new elasticity element made for enforcing weak stress symmetry, Mathematics of Computation 79 (2010), no. 271, 1331-1349.

24. Bernardo Cockburn, Jayadeep Gopalakrishnan, and Raytcho Lazarov, Unified hybridization of discontinuous Galerkin, mixed, and continuous Galerkin methods for second order elliptic problems, SIAM Journal on Numerical Analysis 47 (2009), no. 2, 1319-1365.

25. Blanca Ayuso de Dios, Ivan Georgiev, Johannes Kraus, and Ludmil Zikatanov, A subspace correction method for discontinuous galerkin discretizations of linear elasticity equations, ESAIM: Mathematical Modelling and Numerical Analysis 47 (2013), no. 5, 1315-1333.

26. Shihua Gong, Shuonan $\mathrm{Wu}$, and Jinchao $\mathrm{Xu}$, Mixed finite elements of any order in any dimension for linear elasticity with strongly symmetric stress tensor, arXiv preprint arXiv:1507.01752 (2015).

27. Jayadeep Gopalakrishnan, A Schwarz preconditioner for a hybridized mixed method, Computational Methods in Applied Mathematics Comput. Methods Appl. Math. 3 (2003), no. 1, 116-134.

28. Jayadeep Gopalakrishnan and Johnny Guzmán, Symmetric nonconforming mixed finite elements for linear elasticity, SIAM Journal on Numerical Analysis 49 (2011), no. 4, 1504-1520.

29. Jayadeep Gopalakrishnan and Shuguang Tan, A convergent multigrid cycle for the hybridized mixed method, Numerical Linear Algebra with Applications 16 (2009), no. 9, 689-714.

30. Johnny Guzmán, A unified analysis of several mixed methods for elasticity with weak stress symmetry, Journal of Scientific Computing 44 (2010), no. 2, 156-169.

31. Qingguo Hong, Johannes Kraus, Jinchao Xu, and Ludmil Zikatanov, A robust multigrid method for discontinuous Galerkin discretizations of stokes and linear elasticity equations, Numerische Mathematik 132 (2016), no. 1, 23-49.

32. Jun $\mathrm{Hu}$, Finite element approximations of symmetric tensors on simplicial grids in $\mathbb{R}^{n}$ : The higher order case, Journal of Computational Mathematics 33 (2015), no. 3, 283-296.

33. _ A new family of efficient conforming mixed finite elements on both rectangular and cuboid meshes for linear elasticity in the symmetric formulation, SIAM Journal on Numerical Analysis 53 (2015), no. 3, 1438-1463.

34. Jun Hu and Zhong-Ci Shi, Lower order rectangular nonconforming mixed finite elements for plane elasticity, SIAM Journal on Numerical Analysis 46 (2007), no. 1, 88-102.

35. Jun Hu and Shangyou Zhang, A family of conforming mixed finite elements for linear elasticity on triangular grids, arXiv preprint arXiv:1406.7457 (2014).

36. _ A family of symmetric mixed finite elements for linear elasticity on tetrahedral grids, Science China Mathematics 58 (2015), no. 2, 297-307.

37. _ Finite element approximations of symmetric tensors on simplicial grids in $\mathbb{R}^{n}$ : The lower order case, Mathematical Models and Methods in Applied Sciences 26 (2016), no. 09, $1649-1669$.

38. Xiaozhe Hu, Shuhong Wu, Xiao-Hui Wu, Jinchao Xu, Chen-Song Zhang, Shiquan Zhang, and Ludmil Zikatanov, Combined preconditioning with applications in reservoir simulation, Multiscale Modeling \& Simulation 11 (2013), no. 2, 507-521.

39. Claes Johnson and Bertrand Mercier, Some equilibrium finite element methods for twodimensional elasticity problems, Numerische Mathematik 30 (1978), no. 1, 103-116.

40. Young-Ju Lee, Jinbiao Wu, and Jinru Chen, Robust multigrid method for the planar linear elasticity problems, Numerische Mathematik 113 (2009), no. 3, 473-496.

41. Binjie Li and Xiaoping Xie, Analysis of a family of HDG methods for second order elliptic problems, Journal of Computational and Applied Mathematics 307 (2016), 37-51. 
42. _ BPX preconditioner for nonstandard finite element methods for diffusion problems, SIAM Journal on Numerical Analysis 54 (2016), no. 2, 1147-1168.

43. Hong-Ying Man, Jun $\mathrm{Hu}$, and Zhong-Ci Shi, Lower order rectangular nonconforming mixed finite element for the three-dimensional elasticity problem, Mathematical Models and Methods in Applied Sciences 19 (2009), no. 01, 51-65.

44. John Morgan and Ridgway Scott, A nodal basis for $C^{1}$ piecewise polynomials of degree $n \geq 5$, Mathematics of Computation 29 (1975), no. 131, 736-740.

45. Mary E Morley, A family of mixed finite elements for linear elasticity, Numerische Mathematik 55 (1989), no. 6, 633-666.

46. Weifeng Qiu and Leszek Demkowicz, Mixed hp-finite element method for linear elasticity with weakly imposed symmetry, Computer Methods in Applied Mechanics and Engineering 198 (2009), no. 47, 3682-3701.

47. Weifeng Qiu, Jiguang Shen, and Ke Shi, An HDG method for linear elasticity with strong symmetric stresses, arXiv preprint arXiv:1312.1407 (2013).

48. Joachim Schöberl, Multigrid methods for a parameter dependent problem in primal variables, Numerische Mathematik 84 (1999), no. 1, 97-119.

49. _ Robust multigrid methods for parameter dependent problems, $\mathrm{PhD}$ dissertation, Johannes Kepler Universität Linz, 1999.

50. L Ridgway Scott and Michael Vogelius, Norm estimates for a maximal right inverse of the divergence operator in spaces of piecewise polynomials, RAIRO-Modélisation mathématique et analyse numérique 19 (1985), no. 1, 111-143.

51. L Ridgway Scott and Shangyou Zhang, Finite element interpolation of nonsmooth functions satisfying boundary conditions, Mathematics of Computation 54 (1990), no. 190, 483-493.

52. S-C Soon, B Cockburn, and Henryk K Stolarski, A hybridizable discontinuous Galerkin method for linear elasticity, International journal for numerical methods in engineering $\mathbf{8 0}$ (2009), no. 8, 1058-1092.

53. Andrea Toselli and Olof Widlund, Domain decomposition methods: algorithms and theory, vol. 34, Springer, 2005.

54. Jinchao $\mathrm{Xu}$, Iterative methods by space decomposition and subspace correction, SIAM review 34 (1992), no. 4, 581-613.

55. S-Y Yi, Nonconforming mixed finite element methods for linear elasticity using rectangular elements in two and three dimensions, Calcolo 42 (2005), no. 2, 115-133.

56. Son-Young Yi, A new nonconforming mixed finite element method for linear elasticity, Mathematical Models and Methods in Applied Sciences 16 (2006), no. 07, 979-999.

57. Shangyou Zhang, A new family of stable mixed finite elements for the $3 D$ Stokes equations, Mathematics of computation $\mathbf{7 4}$ (2005), no. 250, 543-554.

Beijing International Center for Mathematical Research, Peking University, BeiJING 100871, P. R. ChINA

E-mail address: gongshihua@pku.edu.cn

Department of Mathematics, Pennsylvania State University, University Park, PA, 16802, USA

E-mail address: sxw58@psu.edu

Department of Mathematics, Pennsylvania State University, University Park, PA 16802, USA

E-mail address: xu@math.psu.edu 